TRANSACTIONS OF THE

AMERICAN MATHEMATICAL SOCIETY

Volume 360, Number 5, May 2008, Pages 2581-2614

S 0002-9947(07)04356-5

Article electronically published on November 28, 2007

\title{
ON ASYMPTOTIC STABILITY IN 3D OF KINKS FOR THE $\phi^{4}$ MODEL
}

\author{
SCIPIO CUCCAGNA
}

\begin{abstract}
We add to a kink, which is a 1 dimensional structure, two transversal directions. We then check its asymptotic stability with respect to compactly supported perturbations in 3D and a time evolution under a Nonlinear Wave Equation (NLW). The problem is inspired by work by Jack Xin on asymptotic stability in dimension larger than 1 of fronts for reaction diffusion equations. The proof involves a separation of variables. The transversal variables are treated as in work on Nonlinear Klein Gordon Equation (NLKG) originating from Klainerman and from Shatah in a particular elaboration due to Delort et al. The longitudinal variable is treated by means of a result by Weder on dispersion for Schrödinger operators in 1D.
\end{abstract}

\section{$\S 1$. INTRODUCTION}

Set $\vec{x}=(x, y) \in \mathbb{R} \times \mathbb{R}^{2}, \Delta$ the full Laplacian, and $\Delta_{y}$ the Laplacian in the variables $y$. We consider the NLW

$$
u_{t t}-\Delta u-u+u^{3}=0,(t, \vec{x}) \in \mathbb{R} \times \mathbb{R}^{n}, n=3,
$$

and the kink solution $\operatorname{th}(x)=\tanh \left(2^{-\frac{1}{2}} x\right)$. We consider solutions of the form

$$
u(t, x, y)=\operatorname{th}(x)+w(t, x, y),
$$

with initial data (chosen real valued not to complicate notation)

$$
w(0, x, y)=w_{0}(x, y) \quad, w_{t}(0, x, y)=w_{1}(x, y) .
$$

We prove:

Theorem 1.1. Consider equation (1.1) with solution (1.2) with initial data (1.3). Assume that the $w_{j}(x, y)$ are smooth, supported in $|x|^{2}+|y|^{2} \leq K^{2}$, for $K>0$ some fixed constant. Then there exists $\epsilon_{0}$ such that if $s=24$ and if $\left(w_{0}, w_{1}\right) \in$ $H^{s}\left(\mathbb{R}^{3}\right) \times H^{s-1}\left(\mathbb{R}^{3}\right)$ with norm smaller than $\epsilon_{0}$, then the solution of the form (1.2) is globally defined in time and such that

$$
|w(t, \vec{x})| \sqrt{1+t} \sqrt{1+|t-| y||}<\infty .
$$

Orbital stability of kinks for $n=1$ is proved in [HPW]; see also the remark on p. 188 of [GSS]. A result similar to Theorem 1.1 can be proved for traveling kinks. For $n=2$ and especially for $n=1$ a similar result is open while for $n \geq 4$, in

Received by the editors June 28, 2004 and, in revised form, November 30, 2005 and March 5, 2006 .

2000 Mathematics Subject Classification. Primary 35L70, 37K40, 35B40.

This research was fully supported by a special grant from the Italian Ministry of Education, University and Research. 
particular for $n \geq 5$, it should be easier to prove. When we replace $u_{t t}$ with $i u_{t}$, we obtain an integrable Schrödinger equation, but (1.1) cannot be treated with the Inverse Scattering Transform, [AS], p. 38. In the case of the heat equation stronger results than ours are well known: for $n=1$ see [H]; for $n>1$ see for $n \geq 4$ [X], for $n=2,3[\mathrm{LX}]$ and for all $n>1[\mathrm{Ka}]$. For the heat equation the fact that most of the spectrum is strictly negative is very helpful, while for (1.1) one can think of the whole spectrum in the imaginary axis. In fact for the heat equation when $n=1$ one exploits that 0 is an isolated eigenvalue and all the rest are strictly negative. For $n \geq 2$ the point 0 is not isolated in the spectrum and so to some extent the transversal variables complicate the spectral picture. At least in part this extra difficulty must be purely formal since, for perturbations localized also in the transversal variables, which only contributes to their smallness, relaxation to a kink must only be more likely. This is the view we take in this paper. Since we do not know how to solve the $n=1$ case we add some extra variables and exploit the dispersion they provide. The case $n=3$ leads to equations with a long range nonlinearity.

The equation for the perturbation can be written

$$
w_{t t}-\Delta w-w+3 \tanh ^{2}\left(2^{-\frac{1}{2}} x\right) w+3 \tanh \left(2^{-\frac{1}{2}} x\right) w^{2}+w^{3}=0 .
$$

We can rewrite

$$
\begin{gathered}
H=-\frac{d^{2}}{d x^{2}}-3 \cosh ^{-2}\left(2^{-\frac{1}{2}} x\right) \\
w_{t t}+H w-\Delta_{y} w+2 w+3 \tanh \left(2^{-\frac{1}{2}} x\right) w^{2}+w^{3}=0 .
\end{gathered}
$$

Recall that the eigenvalues of the operator $-\frac{\hbar^{2}}{\mu} \frac{d^{2}}{d x^{2}}-\frac{V_{0}}{\cosh ^{2}\left(2^{-\frac{1}{2}} x / a\right)}$ are by [GK] given by the formula

$$
\lambda_{n}=-\frac{\hbar^{2}}{\mu a^{2}}\left[\frac{1}{2} \sqrt{\frac{8 \mu V_{0} a^{2}}{\hbar^{2}}+1}-\left(n+\frac{1}{2}\right)\right]^{2}
$$

with $n$ varying among nonnegative integers so that $\frac{1}{2} \sqrt{\frac{8 \mu V_{0} a^{2}}{\hbar^{2}}+1}-\left(n+\frac{1}{2}\right)>0$. In our case $H$ has exactly two eigenvalues, given for $n=0,1$ by

$$
\lambda_{n}=-\frac{1}{2}\left[\frac{1}{2} \sqrt{24+1}-\left(n+\frac{1}{2}\right)\right]^{2}=-\frac{1}{2}(2-n)^{2} .
$$

In particular $\lambda_{0}=-2$ and $\lambda_{1}=-1 / 2$. Notice $\lambda_{2}=0$ is a resonance but not an eigenvalue, that is, we have equality (1.7) with a function bounded but not in $L^{2}(\mathbb{R})$. That 0 is a resonance is used here crucially; see $\S 2$ and the proof of Lemma 12.5. We have

$$
\begin{aligned}
& (H+2) \cosh ^{-2}\left(2^{-\frac{1}{2}} x\right)=0, \\
& \left(H+\frac{1}{2}\right) \cosh ^{-2}\left(2^{-\frac{1}{2}} x\right) \sinh \left(2^{-\frac{1}{2}} x\right)=0, \\
& H\left(\cosh ^{-2}\left(2^{-\frac{1}{2}} x\right)-2 \tanh ^{2}\left(2^{-\frac{1}{2}} x\right)\right)=0 .
\end{aligned}
$$

Following Xin $[\mathrm{X}],[\mathrm{LX}]$ and Kapitula $[\mathrm{Ka}]$ we write the solution in the form

$$
u(t, x, y)=\operatorname{th}(x-\sigma(t, y))+v(t, x-\sigma(t, y), y), \quad \int v(t, x, y) \operatorname{th}^{\prime}(x) d x=0
$$


In turn, if we set

$$
\tilde{\phi}(x)=\sinh \left(2^{-\frac{1}{2}} x\right) \cosh ^{-2}\left(2^{-\frac{1}{2}} x\right), \phi(x)=\frac{\tilde{\phi}(x)}{\|\tilde{\phi}(\cdot)\|_{2}},
$$

we get

$$
v(t, x, y)=\phi(x) a(t, y)+\psi(t, x, y),
$$

with $\psi$ corresponding to the continuous spectrum of $H$. In this paper we prove that locally in space $\psi(t, x, y)=O\left(t^{-\frac{3}{2}+\delta}\right)$ for $\delta>0$ small preassigned, $a(t, y)=O\left(t^{-1}\right)$ and $\sigma(t, y)=O\left(t^{-\frac{1}{2}+\delta}\right)$. Probably these estimates are valid uniformly in space. In the particular case when for the data in (1.3) we have $w_{0}(-x, y)=-w_{0}(x, y)$ and $w_{1}(-x, y)=-w_{1}(x, y)$, where $\sigma(t, y)=0$ for all $t, y$, we are able to prove uniform estimates, but we do not include the argument here.

When we plug ansatz (1.8) into (1.1) we obtain a system of two NLKG equations, one for $\psi$ and one for $a$, and a nonlinear wave equation with zero mass for $\sigma$. The nonlinearity consists of pure powers, including quadratic, and null forms. The equation for $\psi$ has inhomogeneities that depend on the longitudinal variable $x$. If the space dimension $n$ is sufficiently large, then the theory in Shatah [Sh1] and dispersion theory for the linearized operator, Weder [We], lead to the expected result by means of $L^{p} L^{q}$ estimates. If $n$ is small $L^{p} L^{q}$ estimates are not sufficient to close the inequalities. The literature offers as additional tools Klainerman's $[\mathrm{K}]$ vector fields and Shatah's [Sh2] normal forms. In low dimension $n \leq 2$ the two tools are used in conjunction; see $[\mathrm{D}],[\mathrm{DFX}],[\mathrm{GP}],[\mathrm{Ko}],[\mathrm{OTT}]$ and therein for additional references. The methods $[\mathrm{K}]$, [Sh2] are designed for translation invariant equations. In $[\mathrm{K}]$ a translation invariant NLKG is viewed essentially as an ODE in the radial variable in spacetime. In $[\mathrm{D}],[\mathrm{DFX}]$ the approach in $[\mathrm{K}]$ is adapted directly to the nonlinear problem.

Since the inhomogeneities in our system depend only on the longitudinal variable $x$, we implement the method of $[\mathrm{K}],[\mathrm{DFX}]$ (change of coordinates, normalization of the unknowns and energy estimates on hyperboloids using Klainerman's vector fields) only in the variables $(t, y)$, while in the $x$ variable the differentiation needed for energy estimates is by means of the Schrödinger operator $H$. The variables $(t, y)$ are changed into new ones denoted $T, Y$ while $x$ is left alone. Following [D], $[D F X]$ we consider energy estimates and enter the information in the nonlinear system. In [DFX] this leads to a simple system of ODE's for the radial variable $T$ plus small integrable perturbations. Then $L^{\infty}$ estimates are obtained directly from the ODE's using standard ODE methods, for instance standard method of normal forms. Similarly, here we obtain a system of one Klein Gordon equation in $\psi$ with time $T$ and space variable $x$ and an ODE in $T$ for $a$, while it is more convenient to think of the equation for $\sigma$ as an NLW. By variation of parameters and using work by Weder [We], we obtain nice estimates for $\psi$, so that $\psi$ can be eliminated from the system. Now we have reduced to an NLW for $\sigma$ and an ODE for $a$. Thanks to the estimates on $\psi$, by the Morawetz vector field we get nice estimates for $\sigma$. Eventually, up to lower order terms, we have in effect just a closed nonlinear scalar ODE in $a$, with quadratic nonlinearity in $a$, up to a lower order error. We estimate $a$ applying normal forms as in [DFX].

We want to point to two features of the problem. The first, which is certainly fundamental, is the fact that, both here and in [Ka], the problem can be solved only because the nonlinearities are of a specific form, that is, pure power terms 
and (see [Ho], [So]) Klainerman's null forms. The second feature, maybe not as fundamental but just as important for our argument, is that the endpoints result for $p=1, \infty$ in Theorem 2.2, more precisely the dispersive $L^{1} \rightarrow L^{\infty}$ estimate for linear Klein Gordon equations in Corollary 2.3, are crucial. Now, the $L^{1} \rightarrow L^{\infty}$ estimate to our knowledge is known to hold only with Schrödinger operators which, like our linearization $H$ defined under (1.4), have 0 as a resonance and have a transition coefficient satisfying $T(0)=1$; see Lemma 2.1 below. The $L^{1} \rightarrow L^{\infty}$ estimate seems essential because, by the dimension 2 of the $Y$ space, at some point we get an undesired $\log T$ growth factor in the upper bound on $\psi$; see Lemma 11.2. The estimates for $\sigma$ are tight and this $\log T$ term risks to derail all the estimates. Fortunately we are able to prove that the crucial term involved in the estimate for $\sigma$ (see (12.1)) does not have the additional factor. The proof makes use of the $L^{1} \rightarrow L^{\infty}$ estimate in Corollary 2.3. A limitation in the proof is that the $\delta>0$ of say $\psi(t, x, y)=O\left(t^{-\frac{3}{2}+\delta}\right)$ affects the size of the neighborhood of th for initial data, not only the constant in the big $O$.

This paper is structured as follows. In $\S 2$ we state a framework from Kapitula $[\mathrm{Ka}]$ which is necessary to introduce the modulation equations. Then we state results in [We] which give us estimates on groups associated to $H$. In $\S 3$ we "modulate" following [Ka]. This leads us to a system whose nonlinearity is of the right type, in particular displays null form dependence in $\sigma$. All sections from 4 on are heavily dependent on [DFX]. In particular we often state without proof formulas and lemmas which are proved in [DFX]. In $\S 5$ we introduce Klainerman's vectorfields and introduce new coordinates. In a subsection we derive, using the Morawetz vectorfield, various formulas for $\sigma$. In $\S 6$ we describe the basic continuity argument used to prove the main theorem; the rest of the paper consists of the proof that inequalities (6.1) imply the improved inequality (6.2). The crux of the paper starts from $\S 7$, where we first restate the system, formulas (7.1)-(7.2) and (7.5), and we start a long list of multilinear estimates. We advise the reader to skip these estimates at a first reading. In $\S 8$ we prove a high energy estimate, Lemma 8.1, using the material in $\S 7$. At a first reading we advise the reader to read the statement of Lemma 8.1 and skip the rest of the section. In $\S 9$ we rewrite the system emphasizing the variables $x$ and $T$. In $\S 10$ we derive improved low energy estimates for $\mathcal{A}$. In $\S 11$ we derive dispersive estimates for $\Psi$. At the end of $\S 11$ we exploit the estimates in Lemma 8.6, which are derived from the finite speed of propagation. In $\S 12$ we prove the estimates for $\Sigma$, stated in Lemmas 12.2 and 12.4. At a first reading we advise the reader to read the statements of these lemmas, skip all the rest of $\S 12$ and read the closure of the estimates in $\S 13$.

\section{$\S 2$. Spectral DeComposition and LONGitudinal Dispersion}

We start with $\S 2[\mathrm{Ka}]$. We denote by \langle\rangle$_{x}$ the $L^{2}$ inner product in $x . \mathcal{S}(\mathbb{R})$ is the space of rapidly decreasing and smooth functions defined in $\mathbb{R}$. Then, proceeding as in Lemma $2.1[\mathrm{Ka}]$, we have:

Lemma 2.0. Let $\varphi \in \mathcal{S}(\mathbb{R})$. For any $U(x, y), x \in \mathbb{R}, y \in \mathbb{R}^{2}$, let $\langle\varphi, U\rangle_{x}$ be the function in $y$ obtained by taking the inner product in the variable $x \in \mathbb{R}$. Then we have for all integers $k$ and for all $p \in[1, \infty]$ :

$$
\begin{aligned}
& \left\|\langle\varphi, U\rangle_{x}\right\|_{W^{k, p}\left(\mathbb{R}^{2}\right)} \leq\|\varphi\|_{L^{\frac{p}{p-1}(\mathbb{R})}}\|U\|_{W^{k, p}\left(\mathbb{R}^{3}\right)}, \\
& \left\|\varphi(x)\langle\varphi, U\rangle_{x}\right\|_{W^{k, p}\left(\mathbb{R}^{3}\right)} \leq\|\varphi\|_{W^{k, p}(\mathbb{R})}\|\varphi\|_{L^{\frac{p}{p-1}(\mathbb{R})}}\|U\|_{W^{k, p}\left(\mathbb{R}^{3}\right)} .
\end{aligned}
$$


In correspondence to the spectral decomposition of $H$, the identity operator in $L^{2}(\mathbb{R})$, in fact $L^{p}(\mathbb{R})$ for any $p \in[1, \infty]$, can be decomposed into

$$
\mathcal{I}=P_{0}+P_{1}+P_{c}
$$

with $P_{0}$ the projection associated to (1.5), $P_{1}$ the projection associated to (1.6) and $P_{c}$ the projection on the continuous spectrum of $H$. Since $\operatorname{th}^{\prime}(x)$ and $\phi(x)$ are in $\mathcal{S}(\mathbb{R})$, by Lemma 2.0 projections $P_{0}, P_{1}, P_{c}$, and the corresponding splitting (2.0) extend to $W^{k, p}\left(\mathbb{R}^{3}\right)$ for any $p \in[1, \infty]$ and any $k$.

We collect a few technical facts on operator $H$ needed later in the proof. We have:

Lemma 2.1. For the transmission coefficient of $H$, we have $T(0)=1$.

By $(2.12)$ in [We] we have $T(0)=\frac{2 a}{2+a^{2}}$ where $a=\lim _{x \rightarrow-\infty} f_{1}(x, 0)$, with $f_{1}(x, 0)$ the Jost function satisfying $\lim _{x \rightarrow+\infty} f_{1}(x, 0)=1$. If there is a bounded solution $u$ of $H u=0$, by elementary ODE arguments $u$ must be a multiple of $f_{1}(x, 0)$. Then the function in (1.7) appropriately normalized gives $f_{1}(x, 0)$, and since it is even, we have $a=1$.

Lemma 2.1 is very useful because the main result in Weder [We] implies:

Theorem 2.2. Consider for $\varphi \in C_{0}^{\infty}(\mathbb{R})$ the operator $W \varphi=\lim _{t \rightarrow+\infty} e^{i H t} e^{i \frac{d^{2}}{d x^{2}} t} \varphi$. Then $W$ extends into a bounded one to one map from $W^{k, p}(\mathbb{R})$ into itself $\forall p \in$ $[1, \infty]$ and $\forall k \in \mathbb{Z}$, with image given by $P_{c}(H) W^{k, p}(\mathbb{R})$.

If 0 was not a resonance, so that $T(0)=0$, or if $T(0) \neq 1$, we would miss $p=1, \infty$ in Theorem 2.2; see [We]. These endpoints cases are used in Lemma 12.5.

For $P_{c}(H)$ as in Theorem 2.2 set $B=\sqrt{H+2} P_{c}(H)$. Since $W f(H) P_{c}(H)=$ $f\left(-\frac{d^{2}}{d x^{2}}\right) W$ for any measurable bounded function $f$, by Theorem $2.2 B$ has the following dispersive properties:

Corollary 2.3. We have that $\forall p \in[2, \infty]$ the operators $\sin (t B)$ and $\cos (t B)$ send $P_{c}(H) W^{k+2-\frac{4}{p}, \frac{p}{p-1}}(\mathbb{R}) \rightarrow P_{c}(H) W^{k, p}(\mathbb{R})$ with norm bounded by $C_{p} t^{-\frac{1}{2}+\frac{1}{p}}$.

We have the following fact; see p. 296 of [T]:

Lemma 2.4. $B$ is an elliptic pseudo differential operator (pdo) of order 1.

Finally we have:

Lemma 2.5. Set $B^{0}=P_{c}(H)$. If $m$ is even (resp.odd) $\forall p \in[2, \infty]$ (resp. $p \in$ $[2, \infty[)$,

$$
\left\|\left[B^{m}, \partial_{x}\right]: W^{m, p} \rightarrow L^{p} \cap L^{\frac{p}{p-1}}\right\|<\infty .
$$

For $m=2 k$ this follows from

$$
\left[(H+2)^{k} P_{c}(H), \partial_{x}\right]=(H+2)^{k}\left[P_{c}(H), \partial_{x}\right]+\left[(H+2)^{k}, \partial_{x}\right] P_{c}(H) .
$$

For $m=2 k-1,\left[B^{m}, \partial_{x}\right]=B\left[B^{m-1}, \partial_{x}\right]+\left[B^{m-1}, \partial_{x}\right] B$ with $\left[B^{m-1}, \partial_{x}\right]$ bounded from $W^{m, p} \rightarrow W^{1, p} \cap W^{1, \frac{p}{p-1}}$ and from $W^{m-1, p} \rightarrow L^{p} \cap L^{\frac{p}{p-1}}$. Since $B$ is bounded from $W^{m, p} \rightarrow W^{m-1, p}$ and from $W^{1, p} \cap W^{1, \frac{p}{p-1}} \rightarrow L^{p} \cap L^{\frac{p}{p-1}}$, we are done. 


\section{§3. Modulation}

We still follow Kapitula [Ka]. We need to justify formula (1.8). For our purposes the following result is sufficient:

Lemma 3.1. Consider $k \in \mathbb{N}$ and $p \in[1, \infty]$ such that $W^{k, p}\left(\mathbb{R}^{3}\right) \subset C^{0}\left(\mathbb{R}^{3}\right) \cap$ $L^{\infty}\left(\mathbb{R}^{3}\right)$. Then there are positive constants $C$ and $\epsilon_{0}$ such that, for any $\left.\epsilon \in\right] 0, \epsilon_{0}[$ and for any $w(x, y) \in W^{k, p}\left(\mathbb{R}^{3}\right)$ of norm less than $\epsilon$, there exists a unique pair $(v(x, y), \sigma(y))$ with: $v(x, y) \in W^{k, p}\left(\mathbb{R}^{3}\right) ; P_{0} v=0 ; \sigma(y) \in W^{k, p}\left(\mathbb{R}^{2}\right) ;$

$$
\operatorname{th}(x)+w(x, y)=\operatorname{th}(x-\sigma(y))+v(x-\sigma(y), y) ;
$$

the norm of $(v(x, y), \sigma(y))$ is less than $C \epsilon$.

The proof, that we sketch now, follows from [Ka], Lemma 2.2. Assuming (3.1) we can write

$$
w(x, y)=v(x-\sigma(y), y)-\sigma(y) \int_{0}^{1} \operatorname{th}^{\prime}(x-s \sigma(y)) d s .
$$

Define

$$
F(\sigma, w)(y)=\left\langle w(\cdot, y), \operatorname{th}^{\prime}(\cdot-\sigma(y))\right\rangle_{x}+\sigma(y) \int_{0}^{1}\left\langle\operatorname{th}^{\prime}(\cdot-s \sigma(y)), \operatorname{th}^{\prime}(\cdot-\sigma(y))\right\rangle_{x} d s .
$$

Then

$$
F \in C^{1}\left(W^{k, p}\left(\mathbb{R}^{2}\right) \times W^{k, p}\left(\mathbb{R}^{3}\right), W^{k, p}\left(\mathbb{R}^{2}\right)\right),
$$

with $F(0,0)=0$ and $F_{\sigma}(0,0)=\left\|\operatorname{th}^{\prime}\right\|_{2}^{2} \mathcal{I}$. We have that $P_{0} v=0$ is equivalent to $F(\sigma, w)=0$. By the Implicit Function Theorem there is a unique continuous map $\sigma=\sigma(w)$ such that $\sigma(0)=0$ and $F(\sigma(w), w)=0$. We then plug this $\sigma$ in (3.2) and we obtain the desired $v$.

Let us write $Q_{0}(f, g)=f_{t} g_{t}-\nabla_{y} f \cdot \nabla_{y} g$. We plug ansatz (1.8) into equation (1.1) and get, renaming $x-\sigma$ by $x$,

$$
\begin{aligned}
& Q_{0}(\sigma, \sigma) \operatorname{th}^{\prime \prime}(x)-\operatorname{th}^{\prime}(x) \square \sigma-v_{x}(x) \square \sigma+\left(3 \operatorname{th} v^{2}+v^{3}\right)(x) \\
& +\left(\square v+2 v+3\left(\operatorname{th}^{2}-1\right) v\right)(x)+Q_{0}(\sigma, \sigma) v_{x x}-2 Q_{0}\left(\sigma, v_{x}\right)=0 .
\end{aligned}
$$

In the frame associated to (2.0), if we write $v(t, x, y)=P_{1} v(t, x, y)+P_{c} v(t, x, y)=$ $\phi(x) a(t, y)+\psi(t, x, y)$, equation (3.3) splits into a system formed by (3.4)-(3.6) below. We will set $\square_{y}=\partial_{t t}-\Delta_{y}$. We first consider $P_{c}(3.3)$ to obtain:

$$
\begin{aligned}
& \left(\square_{y}+B^{2}\right) \psi-P_{c}\left(\psi_{x}+a \phi^{\prime}\right) \square_{y} \sigma+Q_{0}(\sigma, \sigma) P_{c} \psi_{x x}-2 Q_{0}\left(\sigma, P_{c} \psi_{x}\right) \\
& =-P_{c}\left(F_{2}+F_{3}\right)-Q_{0}(\sigma, \sigma) P_{c} \operatorname{th}^{\prime \prime}+2 Q_{0}(\sigma, a) P_{c} \phi^{\prime}-a Q_{0}(\sigma, \sigma) P_{c} \phi^{\prime \prime} \\
& F_{2}(\psi, a)=3 \operatorname{th}(a \phi+\psi)^{2} \\
& F_{3}(\psi, a)=(a \phi+\psi)^{3} .
\end{aligned}
$$

We consider $\langle(3.3), \phi\rangle_{x}$ (from now on we omit the subscript $x$ and write simply \langle\rangle ) and obtain

$$
\begin{aligned}
& \left(\square_{y}+\frac{3}{2}\right) a=-G_{2}-G_{3}-\left\langle\psi, \phi^{\prime \prime}\right\rangle \square \sigma-Q_{0}(\sigma, \sigma)\left\langle\operatorname{th}^{\prime \prime}, \phi\right\rangle \\
& -2 Q_{0}\left(\sigma,\left\langle\psi, \phi^{\prime}\right\rangle\right)-Q_{0}(\sigma, \sigma)\left\langle\psi, \phi^{\prime \prime}\right\rangle+a Q_{0}(\sigma, \sigma)\left\|\phi^{\prime}\right\|_{2}^{2}
\end{aligned}
$$


where $G_{j}=\left\langle F_{j}, \phi\right\rangle$. Notice that $2 Q_{0}(\sigma, a)\left\langle\phi, \phi^{\prime}\right\rangle=0$. We consider $\left\langle(3.3), \operatorname{th}^{\prime}\right\rangle$ and obtain

$$
\begin{aligned}
& \square_{y} \sigma\left(\left\|\operatorname{th}^{\prime}\right\|_{2}^{2}-\left\langle\psi, \operatorname{th}^{\prime \prime}\right\rangle+a\left\langle\phi^{\prime}, \operatorname{th}^{\prime}\right\rangle\right)=H_{2}+H_{3} \\
& +2 Q_{0}\left(\sigma,\left\langle\psi, \operatorname{th}^{\prime \prime}\right\rangle\right)-2 Q_{0}(\sigma, a)\left\langle\phi^{\prime}, \operatorname{th}^{\prime}\right\rangle+Q_{0}(\sigma, \sigma)\left\langle\psi, \operatorname{th}^{\prime \prime \prime}\right\rangle
\end{aligned}
$$

where $H_{j}=\left\langle F_{j}, \operatorname{th}^{\prime}\right\rangle$. In particular

$$
H_{2}(\psi, a)=6 a\left\langle\psi, \operatorname{th} \phi \operatorname{th}^{\prime}\right\rangle+3\left\langle\operatorname{th} \psi^{2}, \operatorname{th}^{\prime}\right\rangle
$$

where $3 a^{2}\left\langle\operatorname{th} \phi^{2}, \operatorname{th}^{\prime}\right\rangle=0$ because $\phi^{2} \operatorname{th}^{\prime}$ is even while th is odd. Notice that the nonlinearities contain $\sigma$ only inside null forms. We will prove:

Theorem 3.2. Under the hypothesis of Theorem 1.1 we have that for any fixed $\delta>0$ there is an $\epsilon_{0}=\epsilon_{0}(\delta)>0$ such that for initial data as in Theorem 1.1 we have for $T(t, y)=\sqrt{1+\left.\left|t^{2}-\right| y\right|^{2} \mid}$

$$
|\psi(t, \vec{x})| T^{\frac{3}{2}-\delta}(t, y)+|a(t, y)| T(t, y)+|\sigma(t, y)| T^{\frac{1}{2}-\delta}(t, y)<\infty .
$$

Having completed the set up, in the rest of the paper we borrow heavily from $[\mathrm{DFX}]$.

\section{$\S 4$. Short TERM BeHAVIOUR}

We first look at equation (1.4) with initial conditions (1.2). In analogy to Proposition 1.1.4 [DFX] and Proposition $1.4[\mathrm{D}]$, we have:

Proposition 4.1. Let $T_{0}>2 K, s \in \mathbb{N}, s \geq 3$. There are $\epsilon_{0}>0$ and $C>0$ such that for any $\epsilon$ with $0<\epsilon<\epsilon_{0}$ and for any $\left(w_{0}, w_{1}\right)$ in a ball of radius $\epsilon$ in $H^{s+1}\left(\mathbb{R}^{3}\right) \times H^{s}\left(\mathbb{R}^{3}\right)$ and supported in $\left\{(x, y):|x|^{2}+|y|^{2} \leq K^{2}\right\}$, problem (1.4) with (1.2) has a unique solution defined in

$$
\left\{(t, y): t \geq 0,(t+2 K)^{2}-|y|^{2} \leq T_{0}^{2}\right\}
$$

continuous in time with values in $H^{s+1}\left(\mathbb{R}^{3}\right)$ and $C^{1}$ with values in $H^{s}\left(\mathbb{R}^{3}\right)$. Moreover

$$
\sum_{|\alpha| \leq s} \int_{\mathbb{R}^{3}}\left|\left(\partial^{\alpha} w\right)\left(\sqrt{T_{0}^{2}+|y|^{2}}, x, y\right)\right|^{2} d x d y \leq C^{2} \epsilon^{2},
$$

and the restriction of $w(t, x, y)$ and its derivatives on the hypersurface $(t+2 K)^{2}-$ $|y|^{2}=T_{0}^{2}$ is supported inside $\left\{\sqrt{|x|^{2}+|y|^{2}} \leq \frac{T_{0}^{2}-K^{2}}{2 K}\right\}$.

We sketch the proof. By taking $\epsilon_{0}$ small, there is a unique solution $w(t) \in$ $L^{\infty}\left(\left[0, t_{0}\right), H^{s+1}\left(\mathbb{R}^{3}\right)\right) \cap C^{0,1}\left(\left[0, t_{0}\right), H^{s}\left(\mathbb{R}^{3}\right)\right)$ for any preassigned $t_{0}$, Theorem 6.4.11, [Ho], which is also in $C^{0}\left(\left[0, t_{0}\right), H^{s+1}\left(\mathbb{R}^{3}\right)\right) \cap C^{1}\left(\left[0, t_{0}\right), H^{s}\left(\mathbb{R}^{3}\right)\right)$, [So], p. 18. For $t_{0}=\frac{T_{0}^{2}-3 K^{2}}{2 K}$, the intersection of (4.1) with the support of $w(t, x, y)$ is contained in $\left\{t \leq t_{0}\right\} \cap\left\{\left(|x|^{2}+|y|^{2}\right)^{\frac{1}{2}} \leq t_{0}+K\right\}$. (4.2) can be obtained by a trace theorem from the standard energy inequality. 


\section{§5. Change of coordinates and Klainerman's vector fields}

Having disposed of the solution in the region below (4.1), we consider system (3.4)-(3.6) in the region above (4.1). Following [K], [D], [DFX], we replace coordinates $(t, x, y) \rightarrow(T, x, Y)$, we introduce in this new system of coordinates Klainerman's vector fields, only for variables $(t, y)$, and we rewrite (3.4)-(3.6) in the new coordinate system.

Set $t+2 K=T \cosh |Y|, y=T Y \frac{\sinh |Y|}{|Y|}$. Introduce Klainerman vector fields

$$
\begin{aligned}
Z_{0} & =y_{1} \partial_{y_{2}}-y_{2} \partial_{y_{1}}=Y_{1} \partial_{Y_{2}}-Y_{2} \partial_{Y_{1}}, \\
Z_{1} & =(t+2 K) \partial_{y_{1}}+y_{1} \partial_{t} \\
& =\left[\frac{Y_{1}^{2}}{|Y|^{2}}+\frac{Y_{2}^{2} \cosh |Y|}{|Y| \sinh |Y|}\right] \partial_{Y_{1}}+\frac{Y_{1} Y_{2}}{|Y|^{2}}\left[1-\frac{|Y| \cosh |Y|}{\sinh |Y|}\right] \partial_{Y_{2}}, \\
Z_{2} & =(t+2 K) \partial_{y_{2}}+y_{2} \partial_{t} \\
& =\frac{Y_{1} Y_{2}}{|Y|^{2}}\left[1-\frac{|Y| \cosh |Y|}{\sinh |Y|}\right] \partial_{Y_{1}}+\left[\frac{Y_{2}^{2}}{|Y|^{2}}+\frac{Y_{1}^{2} \cosh |Y|}{|Y| \sinh |Y|}\right] \partial_{Y_{2}}, \\
Z_{3} & =\partial_{T} .
\end{aligned}
$$

We denote by $\mathcal{E}^{r}$ the set of functions $(T, x, Y) \rightarrow c(T, x, Y)$ such that for any multi-index $I$ and integer $k$ there is a constant $C_{I, k}$ such that

$$
\left|\partial_{x}^{k} Z^{I} c(T, x, Y)\right| \leq C_{I, k} T^{-m} e^{r|Y|}
$$

where $m$ is the number of $Z_{3}$ factors inside $Z^{I}$.

By Lemma 1.2.2 of [DFX] we have, with $b_{j}^{(\ell)}(Y) \in \mathcal{E}^{0}$,

$$
\partial_{Y_{j}}=b_{j}^{(1)}(Y) Z_{1}+b_{j}^{(2)}(Y) Z_{2} .
$$

Fix spherical coordinates $Y=R(\cos \theta, \sin \theta)$. We consider

$$
\partial_{R}=\frac{Y}{|Y|} \cdot \nabla_{Y}, \quad \partial_{\theta}=-Y_{2} \partial_{Y_{1}}+Y_{1} \partial_{Y_{2}}=\tanh (R)\left[-\frac{Y_{2}}{R} Z_{1}+\frac{Y_{1}}{R} Z_{2}\right] .
$$

Set

$$
\begin{aligned}
& \partial_{t}^{2}-\Delta_{y}=P+\frac{2}{T} \partial_{T}, \quad P=\partial_{T}^{2}-\frac{1}{T^{2}} \Delta_{h y p} \quad \text { where } \\
& \Delta_{h y p}=\partial_{R}^{2}+\frac{\cosh R}{\sinh R} \partial_{R}+\frac{1}{\sinh ^{2} R} \partial_{\theta}^{2}=Z_{1}^{2}+Z_{2}^{2}-Z_{0}^{2} .
\end{aligned}
$$

Next, we consider for $s \in \mathbb{N}$ the spaces $H_{Y}^{s}$ with norm, for $I=\left(I_{0}, I_{1}, I_{2}\right)$ and $|I|=\left|I_{0}\right|+\left|I_{1}\right|+\left|I_{2}\right|$,

$$
\|f\|_{H_{Y}^{s}}^{2}=\sum_{|I| \leq s} \int_{\mathbb{R}^{2}}\left|Z^{I} f(Y)\right|^{2} \sinh (R) d R d \theta .
$$

We set $L_{Y}^{2}=H_{Y}^{0}$. We consider for $s \in \mathbb{N}$ the Sobolev spaces $H_{x Y}^{s}=H_{Y x}^{s}$ with norm

$$
\|f\|_{H_{x Y}^{s}}^{2}=\sum_{|I|+m \leq s} \int_{\mathbb{R}^{2}}\left|\partial_{x}^{m} Z^{I} f(Y)\right|^{2} \sinh (R) d R d \theta d x .
$$

We denote $H_{x Y}^{0}$ by $L_{x Y}^{2}$ or $L^{2}$. 
We now consider the null form $Q_{0}$ in the new coordinates. By elementary computation:

$$
Q_{0}(f, g)=f_{T} g_{T}-\frac{1}{T^{2}} f_{R} g_{R}-\frac{1}{T^{2}} \frac{1}{\sinh ^{2}(R)} f_{\theta} g_{\theta} .
$$

As in $[\mathrm{K}]$ we set $(\psi, a, \sigma)=\frac{1}{T}(\Psi, \mathcal{A}, \Sigma)$. Notice that the above normalization leads to weaker results than in $[\mathrm{DFX}]$. Set

$$
\mathcal{Q}_{0}(f, g)=\left(f_{T}-\frac{1}{T} f\right)\left(g_{T}-\frac{1}{T} g\right)-\frac{f_{R} g_{R}}{T^{2}}-\frac{f_{\theta} g_{\theta}}{T^{2} \sinh ^{2}(R)} .
$$

With the above changes of the independent variables and of the unknowns, system (3.4)-(3.6) becomes

$$
\begin{aligned}
\left(P+B^{2}\right) \Psi- & \frac{1}{T} P_{c}\left(\Psi_{x}+\mathcal{A} \phi_{x}\right) P \Sigma+\frac{1}{T^{2}} \mathcal{Q}_{0}(\Sigma, \Sigma) P_{c} \Psi_{x x}-\frac{2}{T} \mathcal{Q}_{0}\left(\Sigma, P_{c} \Psi_{x}\right) \\
= & -\frac{P_{c}}{T} F_{2}(\Psi, \mathcal{A} \phi(x))-\frac{1}{T} Q_{0}(\Sigma, \Sigma) P_{c} \operatorname{th}^{\prime \prime}+\frac{2}{T} \mathcal{Q}_{0}(\Sigma, \mathcal{A}) P_{c} \phi^{\prime} \\
& -\frac{P_{c}}{T^{2}} F_{3}(\Psi, \mathcal{A} \phi(x))-\frac{1}{T^{2}} \mathcal{A} \mathcal{Q}_{0}(\Sigma, \Sigma) P_{c} \phi^{\prime \prime}
\end{aligned}
$$

$$
\begin{aligned}
\left(P+\frac{3}{2}\right) \mathcal{A}= & -\frac{1}{T} G_{2}(\Psi, \mathcal{A})-\frac{1}{T^{2}} G_{3}(\Psi, \mathcal{A})-\frac{1}{T} \mathcal{Q}_{0}(\Sigma, \Sigma)\left\langle\operatorname{th}^{\prime \prime}, \phi\right\rangle-\frac{1}{T}\left\langle\Psi, \phi^{\prime \prime}\right\rangle P \Sigma \\
& -\frac{2}{T} \mathcal{Q}_{0}\left(\Sigma,\left\langle\Psi, \phi^{\prime}\right\rangle\right)-\frac{1}{T^{2}} \mathcal{Q}_{0}(\Sigma, \Sigma)\left\langle\Psi, \phi^{\prime \prime}\right\rangle+\frac{1}{T^{2}} \mathcal{A} \mathcal{Q}_{0}(\Sigma, \Sigma)\left\|\phi^{\prime}\right\|_{2}^{2},
\end{aligned}
$$

$$
\begin{aligned}
(P \Sigma)\left(\left\|\operatorname{th}^{\prime}\right\|_{2}^{2}\right. & \left.-\frac{1}{T}\left\langle\Psi, \operatorname{th}^{\prime \prime}\right\rangle+\frac{1}{T} \mathcal{A}\left\langle\phi^{\prime}, \operatorname{th}^{\prime}\right\rangle\right)=\frac{1}{T} H_{2}(\Psi, \mathcal{A})+\frac{2}{T} \mathcal{Q}_{0}\left(\Sigma,\left\langle\Psi, \operatorname{th}^{\prime \prime}\right\rangle\right. \\
& -\frac{2}{T} \mathcal{Q}_{0}(\Sigma, \mathcal{A})\left\langle\phi^{\prime}, \operatorname{th}^{\prime}\right\rangle+\frac{1}{T^{2}} H_{3}(\Psi, \mathcal{A})+\frac{1}{T^{2}} \mathcal{Q}_{0}(\Sigma, \Sigma)\left\langle\Psi, \operatorname{th}^{\prime \prime \prime}\right\rangle .
\end{aligned}
$$

By Proposition $4.1 w$ is smooth in (4.1) with bounds on the first few derivatives. The same statement holds for $(\Psi, \mathcal{A}, \Sigma)$ by Lemma 2.0 . We will prove:

Theorem 5.1. Let $s_{0}=23$. Fix a small positive number $\delta>0$. Let $\epsilon_{0}>0$ and let $\epsilon$ be any number $\epsilon \in] 0, \epsilon_{0}[$. Consider smooth initial data for (5.6)-(5.8):

$$
\begin{aligned}
& \Psi\left(T_{0}\right)=\Psi_{(0)}, \partial_{T} \Psi\left(T_{0}\right)=\Psi_{(1)}, \mathcal{A}\left(T_{0}\right)=\mathcal{A}_{(0)}, \partial_{T} \mathcal{A}\left(T_{0}\right)=\mathcal{A}_{(1)}, \\
& \Sigma\left(T_{0}\right)=\Sigma_{(0)}, \partial_{T} \Sigma\left(T_{0}\right)=\Sigma_{(1)}
\end{aligned}
$$

which are the traces on $T=T_{0}$ of the solutions with smooth initial data provided by Proposition 4.1. Suppose we have estimates

$$
\left\|\left(\Psi_{(0)}, \mathcal{A}_{(0)}, \Sigma_{(0)}\right)\right\|_{H^{s_{0}}}+\left\|\left(\Psi_{(1)}, \mathcal{A}_{(1)}, \Sigma_{(1)}\right)\right\|_{H^{s_{0}-1}} \leq \epsilon .
$$

Then it is possible to choose $\epsilon_{0}$ such that system (5.6)-(5.8) with initial conditions (1) satisfying (2) has a unique solution in $C^{0}\left(\left[T_{0}, \infty\right), H^{s_{0}}\right) \cap C^{1}\left(\left[T_{0}, \infty\right), H^{s_{0}-1}\right)$ and such that we have

$$
\left(T^{\frac{1}{2}-\delta} \Psi(T), \mathcal{A}(T), T^{-\frac{1}{2}-\delta} \Sigma(T)\right) \in L_{x Y}^{\infty} .
$$

The hypothesis that the initial data are traces of solutions from Proposition 4.1 is used later when we need to exploit the finite speed of propagation of the solution $w$ of (1.4). 
In the region of existence the solution $(\Psi, \mathcal{A}, \Sigma)$ is smooth. For $q \in \mathbb{N}$, set

$$
\Psi_{q}=\left(Z^{I} B^{m} \Psi\right)_{m+|I| \leq q}, \mathcal{A}_{q}=\left(Z^{I} \mathcal{A}\right)_{|I| \leq q},(\Psi, \mathcal{A})_{q}=\left(\Psi_{q}, \mathcal{A}_{q}\right) .
$$

Similarly set $\Sigma_{q}=\left(Z^{I} \Sigma\right)_{|I| \leq q}$. We state:

Lemma 5.2. We have:

(1) $\left[Z_{j}, P\right]=0$ for $j=0,1,2$; see $[\mathrm{DFX}]$, Lemma 1.2.3;

(2) $\left[Z_{j}, \partial_{T}\right]=0$ for all $j$; see $[\mathrm{DFX}]$, (1.2.10);

(3) $\left[Z_{j}, \Delta_{\text {hyp }}\right]=0$ for all $j$, from the above two claims;

(4) $\left[\partial_{T}, P\right]=\left[\partial_{T},-\frac{1}{T^{2}} \Delta_{h y p}\right]=\frac{2}{T^{3}} \Delta_{h y p}$.

Set $[Z, \mathcal{B}](u, v)=Z \mathcal{B}(u, v)-\mathcal{B}(Z u, v)-\mathcal{B}(u, Z v)$ for any vectorfield $Z$ and bilinear form $\mathcal{B}$. By elementary computations, using formulas $Q_{0}(f, g)=\square(f g)-g \square f-f \square g$ and $\mathcal{Q}_{0}(f, g)=P(f g)-g P f-f P g$ we get:

Lemma 5.3. Consider a multiindex $I$. In view of Lemma 5.2 we can write $Z^{I}=$ $Z_{3}^{k} Z^{I^{\prime}}$, where $Z^{I^{\prime}}$ does not contain $Z_{3}=\partial_{T}$ among its factors. We also set $\mathcal{Q}_{1}=$ $T^{2} \mathcal{Q}_{0}$. The we have:

(1) $\left[Z^{I^{\prime}}, \mathcal{Q}_{0}\right]=0$;

(2) $\left[Z^{I^{\prime}}, \mathcal{Q}_{1}\right]=0$

(3) $\left[T \partial_{T}, \mathcal{Q}_{1}\right]=0$

(4) $\left[T \partial_{T}, \mathcal{Q}_{0}\right]=-2 \mathcal{Q}_{0}$

$\S 5.1$. The use of Morawetz vectorfield. Lemmas 5.5 and 5.6 are crucial for the dispersive estimates for $\sigma$ derived in $\S 12$. In particular we will first derive Lemma 5.5, which is later used in Lemma 12.2. Then we will complement Lemma 5.5 with Lemma 5.6, obtained through a simple computation and leading to the estimates of Lemma 12.4.

To discuss Lemma 5.5 we introduce the Morawetz vectorfield

$$
K_{0}=\left(t^{2}+|y|^{2}\right) \partial_{t}+2 t y \cdot \nabla_{y}+t=T^{2} \cosh (|Y|) \partial_{T}+T \sinh (|Y|) \partial_{R}+T \cosh (|Y|) \text {. }
$$

We write

$$
K_{0} \frac{\Sigma}{T}=\frac{1}{T} \mathcal{K} \Sigma \quad \text { with } \quad \mathcal{K}=T^{2} \cosh (R) \partial_{T}+T \sinh (R) \partial_{R}
$$

Lemma 5.4. We have

$$
\sinh (R)(\mathcal{K} u)(P u)=\partial_{T} \mathcal{P}^{0}+\partial_{R} \mathcal{P}^{1}+\partial_{\theta} \mathcal{P}^{2}
$$

where

$$
\begin{aligned}
& \mathcal{P}^{0}=\frac{1}{2} \sinh (R) \cosh (R)\left[T^{2} u_{T}^{2}+2 T \tanh (R) u_{T} u_{R}+u_{R}^{2}+\frac{u_{\theta}^{2}}{\sinh ^{2}(R)}\right], \\
& \mathcal{P}^{1}=\frac{1}{2}\left[-T u_{T}^{2}-\frac{2}{\tanh (R)} u_{T} u_{R}-\frac{1}{T} u_{R}^{2}+\frac{u_{\theta}^{2}}{T \sinh ^{2}(R)}\right] \sinh ^{2}(R), \\
& \mathcal{P}^{2}=-T^{-1} u_{R} u_{\theta}-\tanh ^{-1}(R) u_{T} u_{\theta} .
\end{aligned}
$$

Proof. With a change of coordinates $(t, y) \rightarrow(T, Y)$ and for $f=T^{-1} u$, we get $d t d y\left(K_{0} f\right) \square_{t y} f=d T d R d \theta \sinh (R) T\left(T \cosh (R) u_{T}+\sinh (R) u_{R}\right) P u$.

We have

(1)

$$
T^{2} \sinh (R) \cosh (R) u_{T} u_{T T}=\left(\frac{T^{2}}{2} \sinh (R) \cosh (R) u_{T}^{2}\right)_{T}-T \sinh (R) \cosh (R) u_{T}^{2} .
$$


We have

$$
\begin{aligned}
T \sinh ^{2}(R) u_{R} u_{T T} & =\left(T \sinh ^{2}(R) u_{R} u_{T}\right)_{T}-\sinh ^{2}(R) u_{R} u_{T}-T \sinh ^{2}(R) u_{R T} u_{T} \\
& =\left(T \sinh ^{2}(R) u_{R} u_{T}\right)_{T}-\sinh ^{2}(R) u_{R} u_{T} \\
& -\left(\frac{T}{2} \sinh ^{2}(R) u_{T}^{2}\right)_{R}+T \sinh (R) \cosh R u_{T}^{2} .
\end{aligned}
$$

We have

$$
\begin{aligned}
& -\sinh (R) \cosh (R) u_{T} u_{R R}=-\left(\sinh (R) \cosh (R) u_{T} u_{R}\right)_{R} \\
& +\frac{1}{2}\left(\sinh (R) \cosh (R) u_{R}^{2}\right)_{T}+\left(\cosh ^{2}(R)+\sinh ^{2}(R)\right) u_{T} u_{R} .
\end{aligned}
$$

We have

(4) $-\frac{1}{T} \sinh ^{2}(R) u_{R} u_{R R}=-\frac{1}{2}\left(\frac{1}{T} \sinh ^{2}(R) u_{R}^{2}\right)_{R}+\frac{1}{T} \sinh (R) \cosh (R) u_{R}^{2}$.

Another term is

$$
-\cosh ^{2}(R) u_{T} u_{R}
$$

Another term is

$$
-\frac{1}{T} \sinh (R) \cosh (R) u_{R}^{2}
$$

We have

$$
-\tanh ^{-1}(R) u_{T} u_{\theta \theta}=-\left(\tanh ^{-1}(R) u_{T} u_{\theta}\right)_{\theta}+\frac{1}{2}\left(\tanh ^{-1}(R) u_{\theta}^{2}\right)_{T} .
$$

We have

$$
-T^{-1} u_{R} u_{\theta \theta}=-\left(T^{-1} u_{R} u_{\theta}\right)_{\theta}+\frac{1}{2}\left(T^{-1} u_{\theta}^{2}\right)_{R} .
$$

Adding up the numbered formulas and simplifying we obtain Lemma 5.4.

By Lemma 5.4 we have

$$
\partial_{T}\left(\frac{\mathcal{P}^{0}}{\cosh (R)}\right)+\partial_{R}\left(\frac{\mathcal{P}^{1}}{\cosh (R)}\right)+\partial_{\theta}\left(\frac{\mathcal{P}^{2}}{\cosh (R)}\right)+\frac{\tanh (R)}{\cosh (R)} \mathcal{P}^{1}=\tanh (R) \mathcal{K} u P u .
$$

By the last formula we have:

Lemma 5.5. Suppose $P u=F(T, Y)$. Then, for $\mathcal{P}^{j}$ as above, we have:

$$
\begin{aligned}
& \partial_{T}\left(\mathcal{P}^{0} / \cosh (R)\right)+\partial_{R}\left(\mathcal{P}^{1} / \cosh (R)\right)+\partial_{\theta}\left(\mathcal{P}^{2} / \cosh (R)\right) \\
& =\sinh (R)\left(T^{2} \partial_{T} u+T \tanh (R) \partial_{R} u\right) F-\frac{\tanh (R)}{\cosh (R)} \mathcal{P}^{1} .
\end{aligned}
$$

To get the desired dispersion for $\sigma$ we will need to supplement Lemma 5.5 with the following elementary computation:

Lemma 5.6. Suppose $P u=F(T, Y)$. Then we have

$$
\begin{aligned}
\sinh (R) T^{2} u_{T} P u & =\left[\frac{\sinh (R)}{2}\left(T^{2} u_{T}^{2}+u_{R}^{2}+\frac{u_{\theta}^{2}}{\sinh ^{2}(R)}\right)\right]_{T} \\
& -\left(\sinh (R) u_{R} u_{T}\right)_{R}-\left(\sinh ^{-1}(R) u_{\theta} u_{T}\right)_{\theta}-T \sinh (R) u_{T}^{2} .
\end{aligned}
$$


The proof follows from a direct computation. Here notice

$$
\begin{aligned}
& \sinh (R) T^{2} u_{T} u_{T T}=\frac{1}{2}\left(\sinh (R) T^{2} u_{T}^{2}\right)_{T}-T \sinh (R) u_{T}^{2}, \\
& -\sinh (R) u_{T} u_{R R}=-\left(\sinh (R) u_{T} u_{R}\right)_{R}+\cosh (R) u_{T} u_{R}+\frac{1}{2}\left(\sinh (R) u_{R}^{2}\right)_{T}, \\
& -\sinh ^{-1}(R) u_{T} u_{\theta \theta}=-\left(\sinh ^{-1}(R) u_{T} u_{\theta}\right)_{\theta}+\frac{1}{2}\left(\sinh ^{-1}(R) u_{\theta}^{2}\right)_{T} .
\end{aligned}
$$

\section{§6. EnERgy AND A CONTINUity ARGUMENT}

We define, for appropriate $f(T, Y)$,

$$
\tilde{E}(T, f)=\int_{\mathbb{R}^{2}}\left[\left|f_{T}\right|^{2}+\left|\frac{\partial_{R}}{T} f\right|^{2}+\left|\frac{1}{T \sinh (R)} \partial_{\theta} f\right|^{2}\right] \sinh (R) d R d \theta .
$$

Next define

$$
\begin{aligned}
& E_{(1)}(T, \Psi)=\int_{\mathbb{R}} \tilde{E}(T, \Psi) d x+\int_{\mathbb{R}^{3}}|B \Psi|^{2} \sinh (R) d R d \theta d x \\
& E_{(2)}(T, \mathcal{A})=\tilde{E}(T, \mathcal{A})+\int_{\mathbb{R}^{2}} \frac{3}{2}|\mathcal{A}|^{2} \sinh (R) d R d \theta .
\end{aligned}
$$

We call $E(T,(\Psi, \mathcal{A}))=E_{(1)}(T, \Psi)+E_{(2)}(T, \mathcal{A})$. We have the following lemma; see [DFX], Lemma 2.1.1:

Lemma 6.1. We have

$$
\begin{aligned}
& \frac{d}{d T} E_{(1)}(T, \Psi) \leq-2 \Re \int_{\mathbb{R}^{3}} \bar{\Psi}_{T}\left(P \Psi+B^{2} \Psi\right) \sinh (R) d R d \theta d x, \\
& \frac{d}{d T} E_{(2)}(T, \mathcal{A}) \leq-2 \Re \int_{\mathbb{R}^{2}} \overline{\mathcal{A}}_{T}\left(P \mathcal{A}+\frac{3}{2} \mathcal{A}\right) \sinh (R) d R d \theta, \\
& \frac{d}{d T} \tilde{E}(T, \Sigma) \leq-2 \Re \int_{\mathbb{R}^{2}} \bar{\Sigma}_{T} P \Sigma \sinh (R) d R d \theta .
\end{aligned}
$$

Next consider $(\operatorname{see}(5.5)) E_{q}(T)=E\left(T,(\Psi, \mathcal{A})_{q}\right)=E_{(1)}\left(T, \Psi_{q}\right)+E_{(2)}\left(T, \mathcal{A}_{q}\right)$ where

$$
E_{(1)}\left(T, \Psi_{q}\right)=\sum_{m+|I| \leq q} E_{(1)}\left(T, B^{m} Z^{I} \Psi\right), E_{(2)}\left(T, \mathcal{A}_{q}\right)=\sum_{|I| \leq q} E_{(2)}\left(T, Z^{I} \mathcal{A}\right) .
$$

Similarly set

$$
\tilde{E}_{q}(T)=\tilde{E}\left(T, \Sigma_{q}\right)=\sum_{|I| \leq q} \tilde{E}\left(T, Z^{I} \Sigma\right) .
$$

We fix integers $N=22$ and $N^{\prime}=14 . N$ and $N^{\prime}$ are chosen so that $N \geq N^{\prime}+8$ and $N^{\prime} \geq\left[\frac{N}{2}\right]+3$ and $N^{\prime} \geq 8$. Next we fix a small number $\delta>0$. We then fix $p \in(2, \infty)$ so that $\delta \geq \frac{2}{p}$ (we pick $p \neq \infty$ because pdo's are not well behaved in $\left.L^{\infty}\right)$. Next, we suppose that in an interval $\left[T_{0}, T^{*}[\right.$ we have

$$
\begin{aligned}
& E\left(T_{0},(\Psi, \mathcal{A})_{N}\right)+\tilde{E}\left(T_{0}, \Sigma_{N}\right)<\epsilon^{2}, \\
& \sup _{T \in\left[T_{0}, T^{*}[\right.}\left(T^{\frac{1}{2}-2 \delta}\left\|\Psi_{N^{\prime}}\right\|_{L_{Y}^{2} L_{x}^{p}}+T^{\frac{1}{2}-\delta} \tilde{E}^{\frac{1}{2}}\left(T, \Sigma_{N^{\prime}}\right)\right) \leq \mu^{\prime} \epsilon, \\
& \sup _{T \in\left[T_{0}, T^{*}[\right.}\left(\left\|\Psi_{N^{\prime}}\right\|_{L_{Y}^{2} L_{x}^{2}}+\left\|\partial_{T} \Psi_{N^{\prime}}\right\|_{L_{Y}^{2} L_{x}^{2}}+\left\|\mathcal{A}_{N^{\prime}}\right\|_{L_{Y}^{2}}+\left\|\partial_{T} \mathcal{A}_{N^{\prime}}\right\|_{L_{Y}^{2}}\right) \leq \mu^{\prime} \epsilon .
\end{aligned}
$$


The crux of the proof consists in the following continuity argument. We need to show that we can choose $\mu^{\prime}$ and $\epsilon\left(\mu^{\prime}\right)$ so that for any $\left.\epsilon \in\right] 0, \epsilon\left(\mu^{\prime}\right)[,(6.1)$ implies

$$
\begin{aligned}
& \sup _{T \in\left[T_{0}, T^{*}[\right.}\left(T^{\frac{1}{2}-2 \delta}\left\|\Psi_{N^{\prime}}\right\|_{L_{Y}^{2} L_{x}^{p}}+T^{\frac{1}{2}-\delta} \tilde{E}^{\frac{1}{2}}\left(T, \Sigma_{N^{\prime}}\right)\right) \leq \frac{\mu^{\prime}}{2} \epsilon \\
& \sup _{T \in\left[T_{0}, T^{*}[\right.}\left(\left\|\Psi_{N^{\prime}}\right\|_{L_{Y}^{2} L_{x}^{2}}+\left\|\partial_{T} \Psi_{N^{\prime}}\right\|_{L_{Y}^{2} L_{x}^{2}}+\left\|\mathcal{A}_{N^{\prime}}\right\|_{L_{Y}^{2}}+\left\|\partial_{T} \mathcal{A}_{N^{\prime}}\right\|_{L_{Y}^{2}}\right) \leq \frac{\mu^{\prime}}{2} \epsilon .
\end{aligned}
$$

Once we have that $(6.1) \Rightarrow(6.2)$, we are done. Indeed, suppose $T^{*}<\infty$. In the region $T \in\left[T_{0}, T^{*}\right.$, by the finite speed of propagation the support of $w(t, x, y)$ is a bounded set. For any point $P_{0}=\left(t_{0}, x_{0}, y_{0}\right)$, on the hypersurface $T=T^{*}$ the behavior of $w(t, x, y)$ depends only on the values of $w(t, x, y)$ in the cone defined by $t_{0}-\epsilon_{1}<t<t_{0}$, for any $\epsilon_{1}>0$ and $\left|(x, y)-\left(x_{0}, y_{0}\right)\right| \leq t_{0}-t$. For $\epsilon_{1}$ small, the cone is in the region $T \in\left[T_{0}, T^{*}[\right.$. On the cone, (6.1) implies that a large number of derivatives of $w(t, x, y)$ is bounded, and so $w(t, x, y)$ does not blow up at $P_{0}$. Therefore we can conclude that there is a $T_{1}>T^{*}$ such that $w(t, x, y)$ can be extended in a $\left[T_{0}, T_{1}[\right.$. By (6.2) we can assume (6.1) is valid in this larger region. This implies that we can choose $T^{*}=\infty$.

\section{$\S 7$. Various inequalities}

We start by rewriting equations (5.3)-(5.5). Set

$$
\Omega=\left\|\operatorname{th}^{\prime}\right\|_{2}^{2}-\frac{1}{T}\left\langle\Psi, \operatorname{th}^{\prime \prime}\right\rangle+\frac{1}{T} \mathcal{A}\left\langle\phi^{\prime}, \operatorname{th}^{\prime}\right\rangle .
$$

Lemma 7.1. There is a a constant $C\left(\mu^{\prime}\right)$ such that $\forall T \in\left[T_{0}, T^{*}[\right.$ we have

$$
\begin{aligned}
& \left\|\Sigma_{N^{\prime}-1}(T)\right\|_{L_{Y}^{\infty}} \leq C\left(\mu^{\prime}\right) \epsilon T^{\frac{1}{2}+\delta},\left\|\partial_{T} \Sigma_{N^{\prime}-2}(T)\right\|_{L_{Y}^{\infty}} \leq C\left(\mu^{\prime}\right) \epsilon T^{-\frac{1}{2}+\delta}, \\
& \left\|\Psi_{N^{\prime}-2}(T)\right\|_{L_{Y}^{\infty} L_{x}^{p}} \leq C\left(\mu^{\prime}\right) \epsilon T^{-\frac{1}{2}+\delta},\left\|\mathcal{A}_{N^{\prime}-2}(T)\right\|_{L_{Y}^{\infty}} \leq C\left(\mu^{\prime}\right) \epsilon
\end{aligned}
$$

by the Sobolev Embedding Theorem; see [DFX] Corollary 2.2.4, and (6.1).

By Lemma 7.1, $\forall T \in\left[T_{0}, T^{*}\left[\right.\right.$ we have $\Omega \approx\left\|\operatorname{th}^{\prime}\right\|_{2}^{2}$ for $\epsilon \ll 1$. We will denote schematically $L(\Psi, \mathcal{A})=\lambda \mathcal{A}+\mu\langle\Psi, \psi\rangle$ (or $L(\Psi, \mathcal{A})=\lambda \mathcal{A} \psi(x)+\mu \Psi)$ for $\lambda$ and $\mu$ constants with $|\lambda|+|\mu| \leq C$ for a fixed $C$, and for $\psi(x)$ some Schwartz function. For $L$ changing from place to place, we can write schematically

$$
\begin{aligned}
& P \Sigma=\frac{\mathcal{H}}{\Omega}, \quad \mathcal{H}=\frac{1}{T} H_{2}(\Psi, \mathcal{A})+\frac{1}{T} \mathcal{Q}_{0}(\Sigma, L(\Psi, \mathcal{A})) \\
& +\frac{1}{T^{2}} H_{3}(\Psi, \mathcal{A})+\frac{1}{T^{2}} \mathcal{Q}_{0}(\Sigma, \Sigma) L(\Psi, \mathcal{A}) .
\end{aligned}
$$

Next, let us write schematically

$$
\begin{aligned}
& \left(P+\frac{3}{2}\right) \mathcal{A}=-\frac{1}{T} G_{2}(\Psi, \mathcal{A})-\frac{1}{T^{2}} G_{3}(\Psi, \mathcal{A})-\frac{1}{T} \mathcal{Q}_{0}(\Sigma, \Sigma)\left\langle\mathrm{th}^{\prime \prime}, \phi\right\rangle \\
& -\frac{1}{T}\left\langle\Psi, \phi^{\prime \prime}\right\rangle \frac{\mathcal{H}}{\Omega}-\frac{2}{T} \mathcal{Q}_{0}(\Sigma, L(\Psi, \mathcal{A}))-\frac{1}{T^{2}} \mathcal{Q}_{0}(\Sigma, \Sigma) L(\Psi, \mathcal{A}) .
\end{aligned}
$$

We now rewrite the equation for $\Psi$. We consider a symmetric matrix with entries

$$
\begin{aligned}
& r_{x x}=\frac{1}{T^{2}} \mathcal{Q}_{0}(\Sigma, \Sigma), \quad r_{x R}=r_{R x}=\frac{2}{T^{3}} \Sigma_{R}, \\
& r_{x T}=r_{T x}=-\frac{2}{T}\left(\Sigma_{T}-\frac{\Sigma}{T}\right) .
\end{aligned}
$$


Next notice

$$
\frac{2 \Sigma_{\theta}}{T^{3} \sinh ^{2}(R)} \Psi_{x \theta}=\frac{2 \Sigma_{\theta}}{T^{3} \sinh (R)} \sum_{j=1}^{2} a_{j}(Y) \Psi_{x Y_{j}},
$$

with $a_{1}(Y)=-\frac{Y_{2}}{\sinh (R)}$ and $a_{2}(Y)=\frac{Y_{1}}{\sinh (R)}$. We set for $j=1,2$

$$
r_{x Y_{j}}=r_{Y_{j} x}=\frac{2 \Sigma_{\theta}}{T^{3} \sinh (R)} a_{j}(Y) .
$$

We then write

$$
\begin{aligned}
& \left(P+B^{2}\right) \Psi+\sum_{\alpha} r_{x \alpha} \partial_{x} \partial_{\alpha} \Psi=-\frac{P_{c}}{T} F_{2}(\Psi, \mathcal{A}) \\
& -\frac{P_{c}}{T^{2}} F_{3}(\Psi, \mathcal{A})+\frac{2}{T} \mathcal{Q}_{0}(\Sigma, \mathcal{A}) P_{c} \phi^{\prime}-\frac{1}{T} \mathcal{Q}_{0}(\Sigma, \Sigma) P_{c} \mathrm{th}^{\prime \prime} \\
& -\frac{1}{T^{2}} \mathcal{A} \mathcal{Q}_{0}(\Sigma, \Sigma) P_{c} \phi^{\prime \prime}+\frac{1}{T}\left(\Psi_{x}+\mathcal{A} P_{c} \phi^{\prime}\right) \frac{\mathcal{H}}{\Omega} .
\end{aligned}
$$

We now start a long list of inequalities on the terms in the right hand side of equations (7.1)-(7.2) and (7.5). We advise the reader to skip the remaining part of this section at a first reading and to come back to these lemmas when they are referenced later.

Lemma 7.2. Assume (6.1) and let $\mathcal{B}_{j}(\Psi, \mathcal{A}), j=2$ (resp. $j=3$ ) be one of $G_{2}(\Psi, \mathcal{A})$ and $H_{2}(\Psi, \mathcal{A})$ (resp. $G_{3}(\Psi, \mathcal{A})$ and $H_{3}(\Psi, \mathcal{A})$ ). Then for $|I| \leq N$ we have

$$
\left\|Z^{I} \mathcal{B}_{j}(\Psi, \mathcal{A})\right\|_{L_{Y}^{2}} \leq C\left(\mu^{\prime}\right) \epsilon\left\|(\Psi, \mathcal{A})_{N}\right\|_{L_{Y}^{2}}
$$

It is an immediate consequence of Lemma 7.1 and of the Leibnitz rule.

Lemma 7.3. Assume (6.1) and let $|I| \leq N^{\prime}$. Then, for $\mathcal{B}_{2}(\Psi, \mathcal{A})$ a quadratic expression of the form $\mathcal{B}_{2}=\mathcal{A}\left\langle\Psi, \varphi_{1}\right\rangle+\left\langle\Psi^{2}, \varphi_{2}\right\rangle$ for $\varphi_{j}(x) \in \mathcal{S}(\mathbb{R})$, we have

$$
\left\|Z^{I} \mathcal{B}_{2}(\Psi, \mathcal{A})\right\|_{L_{Y}^{2}} \leq \frac{C\left(\mu^{\prime}\right) \epsilon^{2}}{T^{\frac{1}{2}-2 \delta}} .
$$

For $\mathcal{B}_{3}\left(\left\langle\Psi, \varphi_{1}\right\rangle, \mathcal{A}\right)$ a cubic expression in the arguments, for $|I| \leq N^{\prime}$ we have

$$
\left\|Z^{I} \mathcal{B}_{3}\left(\left\langle\Psi, \varphi_{1}\right\rangle, \mathcal{A}\right)\right\|_{L_{Y}^{2}} \leq C\left(\mu^{\prime}\right) \epsilon^{3} .
$$

Remark. Notice that $H_{2}(\Psi, \mathcal{A})$ is by the discussion after $(3.6)$ of the above form. Similarly, $G_{2}(\Psi, \mathcal{A})-3 \operatorname{th} \mathcal{A}^{2}$ is of the above form.

The proof of Lemma 7.3 follows from the Leibnitz rule, Lemma 7.1 and (6.1).

Lemma 7.4. Assume (6.1). Then for $|I|+m \leq N$ we have

$$
\left\|Z^{I} B^{m} F_{j}(\Psi, \mathcal{A})\right\|_{2} \leq C\left(\mu^{\prime}\right) \epsilon\left\|(\Psi, \mathcal{A})_{N}\right\|_{2} .
$$

If $|I|+m \leq N^{\prime}$ we have

$$
\left\|Z^{I} B^{m} F_{3}(\Psi, \mathcal{A})\right\|_{L_{Y}^{2} W_{x}^{1, \frac{p}{p-1}}} \leq C\left(\mu^{\prime}\right) \epsilon\left(\epsilon+T^{-\frac{1}{2}+2 \delta}\left\|(\Psi, \mathcal{A})_{N}\right\|_{2}\right)\left\|(\Psi, \mathcal{A})_{N}\right\|_{2} .
$$

We start with $Z^{I} B^{m} F_{2}(\Psi, \mathcal{A})$ which is a sum of terms of the form

$$
\begin{gathered}
{\left[B^{m} C_{J, K}(T, x, Y) \phi^{2}(x)\right]\left(Z^{J} \mathcal{A}(T, Y)\right)\left(Z^{K} \mathcal{A}(T, Y)\right),} \\
B^{m}\left[C_{J, K}(T, x, Y) \phi(x)\left(Z^{J} \mathcal{A}(T, Y)\right)\left(Z^{K} \Psi(T, x, Y)\right)\right], \\
B^{m}\left[C_{J, K}(T, x, Y)\left(Z^{J} \Psi(T, x, Y)\right)\left(Z^{K} \Psi(T, x, Y)\right)\right]
\end{gathered}
$$


with $C_{J, K} \in \mathcal{E}^{0}$ and $|J|+|K| \leq|I|$. Now $B^{m} \circ C_{J, K}=C_{J, K} \circ B^{m}+\left[B^{m}, C_{J, K}\right]$ with $\left[B^{m}, C_{J, K}\right]$ a pdo of order $m-1$ by Lemma 2.4. By Lemma 2.4 we have $\left\|B^{m}(j)\right\|_{2} \leq\|(j)\|_{L_{Y}^{2} H_{x}^{m}}$ for $j=3,4,5$ and so it is enough to show that for linear combinations $L_{j}(\Psi, \mathcal{A})=\lambda_{j} \Psi(T, x, Y)+\mu_{j} \psi(x) \mathcal{A}(T, Y)$, with bounded coefficients, we have

$$
\left.\|\left(Z^{J} \partial_{x}^{j} L_{1}\right)\right)\left(Z^{K} \partial_{x}^{k} L_{2}\right) \|_{2} \leq \operatorname{rhs}(1) .
$$

If say $|K|+k \leq \frac{N}{2}$, then by Sobolev embedding we bound by

$$
\left\|Z^{K} \partial_{x}^{k} L_{2}\right\|_{\infty}\left\|(\Psi, \mathcal{A})_{N}(T)\right\|_{2} \leq C\left\|L_{2}\right\|_{H_{Y}^{|K|+2} W_{x}^{k+1, p}}\left\|(\Psi, \mathcal{A})_{N}(T)\right\|_{2} .
$$

Since $B$ is an elliptic pdo, by $\left[\frac{N}{2}\right]+3 \leq N^{\prime}$ and by $(6.1)$

$$
\left\|L_{2}(T)\right\|_{H_{Y}^{|K|+2} W_{x}^{k+1, p}} \leq C\left\|\left(\lambda_{2} \Psi(T)+\mu_{2} \psi(x) \mathcal{A}(T)\right)_{N^{\prime}}\right\|_{L_{Y}^{2} L_{x}^{p}} \leq C \mu^{\prime} \epsilon ;
$$

hence we have obtained (6). When we prove (1) for $j=3$ we proceed similarly reducing to

$$
\left\|\left(Z^{J} \partial_{x}^{j} L_{1}\right)\left(Z^{K} \partial_{x}^{k} L_{2}\right)\left(Z^{W} \partial_{x}^{w} L_{3}\right)\right\|_{2} \leq \operatorname{rhs}(1),
$$

now with two factors with fewer than $\frac{N}{2}$ derivatives, say $|K|+k \leq \frac{N}{2}$ and $|W|+w \leq$ $\frac{N}{2}$. For either of them we have a bound like (7) and so we have (8).

We turn to the proof of (2). By Lemma 2.4 and using the above notation,

$$
\left\|B^{m}\left(Z^{J} L_{1} Z^{K} L_{2} Z^{W} L_{3}\right)\right\|_{L_{Y}^{2} W_{x}^{1, \frac{p}{p-1}}} \leq C\left\|Z^{J} L_{1} Z^{K} L_{2} Z^{W} L_{3}\right\|_{L_{Y}^{2} W_{x}^{m+1, \frac{p}{p-1}}} .
$$

Terms of the form $\psi(x) \mathcal{A}(\Psi, \psi(x) \mathcal{A})^{2}$ are bounded, by Schwartz and Sobolev inequalities and Sobolev embedding and by $(6.1)$, by

$$
\left\|(\Psi, \psi(x) \mathcal{A})_{N^{\prime}}\right\|_{L_{Y}^{2} L_{x}^{p}}^{2}\left\|(\Psi, \mathcal{A})_{N}(T)\right\|_{2} \leq C\left(\mu^{\prime}\right) \epsilon^{2}\left\|(\Psi, \mathcal{A})_{N}(T)\right\|_{2}
$$

Terms like $\Psi^{3}$ are bounded by

$$
\left\|\Psi_{N^{\prime}}\right\|_{L_{Y}^{2} L_{x}^{p}}\left\|\Psi_{N}(T)\right\|_{2}^{2} \leq \mu^{\prime} T^{-\frac{1}{2}+2 \delta} \epsilon\left\|(\Psi, \mathcal{A})_{N}(T)\right\|_{2}^{2} .
$$

Lemma 7.5. Let $L=L(\langle\Psi, \psi\rangle, \mathcal{A})=\lambda\langle\Psi(T, x, Y), \psi(x)\rangle_{L_{x}^{2}}+\mu \mathcal{A}(T, Y)$ with $\lambda$ and $\mu$ two constants, bounded by a fixed number. Assume (6.1). Then, for $|I| \leq N$ we have $\left\|Z^{I} \mathcal{Q}_{0}(\Sigma, L)\right\|_{2} \leq(7.6)$ with

$$
\frac{C\left(\mu^{\prime}\right) \epsilon^{2}}{T}+C\left(\mu^{\prime}\right) \epsilon\left(E_{N}^{\frac{1}{2}}(T)+\tilde{E}_{N}^{\frac{1}{2}}(T)\right)+\frac{C\left(\mu^{\prime}\right) \epsilon}{T} \int_{T_{0}}^{T}\left(E_{N}^{\frac{1}{2}}(\tau)+\tilde{E}_{N}^{\frac{1}{2}}(\tau)\right) d \tau .
$$

We consider $Z_{3}^{k} Z^{I^{\prime}} \mathcal{Q}_{0}(\Sigma, L)$, where $Z^{I^{\prime}}$ does not contain $Z_{3}$. By Lemma 5.3

$$
Z^{I^{\prime}} \mathcal{Q}_{0}(\Sigma, L)=\sum C_{J^{\prime} K^{\prime}} \mathcal{Q}_{0}\left(Z^{J^{\prime}} \Sigma, Z^{K^{\prime}} L\right)
$$

with $C_{J^{\prime} K^{\prime}}$ constants. Suppose $k=0$. If $\left|J^{\prime}\right| \leq\left[\frac{N}{2}\right]<N^{\prime}-1$, by Lemma 7.1 $\left\|Z^{J^{\prime}} \Sigma(T)\right\|_{L_{Y}^{\infty}} \leq C\left(\mu^{\prime}\right) T^{\frac{1}{2}+\delta} \epsilon$. Consequently by $(6.1)$

$$
\left\|\mathcal{Q}_{0}\left(Z^{J^{\prime}} \Sigma, Z^{K^{\prime}} L\right)(T)\right\|_{L_{Y}^{2}} \leq \frac{C\left(\mu^{\prime}\right) \epsilon}{T^{\frac{1}{2}-\delta}} E_{N}^{\frac{1}{2}}(T)
$$

From this point on, we assume $\left|J^{\prime}\right|>\left[\frac{N}{2}\right]$ and $\left|K^{\prime}\right| \leq\left[\frac{N}{2}\right]$. We claim:

$$
\frac{1}{T^{2}}\left\|\left(\partial_{R} Z^{J^{\prime}} \Sigma\right)\left(\partial_{R} Z^{K^{\prime}} L\right)+\frac{1}{\sinh ^{2}(R)}\left(\partial_{\theta} Z^{J^{\prime}} \Sigma\right)\left(\partial_{\theta} Z^{K^{\prime}} L\right)\right\|_{2} \leq \frac{C\left(\mu^{\prime}\right) \epsilon}{T} \tilde{E}^{\frac{1}{2}}\left(T, \Sigma_{N}\right) \text {. }
$$


To show the claim notice that $\left\|\partial_{R} Z^{K^{\prime}} L\right\|_{\infty} \leq\left\|(\Psi, \mathcal{A})_{\left[\frac{N}{2}\right]+1}\right\|_{\infty}$. Similarly, equality $\partial_{\theta}=\tanh (R)\left[-\frac{Y_{2}}{R} Z_{1}+\frac{Y_{1}}{R} Z_{2}\right]$ implies $\left\|\frac{1}{\sinh (R)} \partial_{\theta} Z^{K^{\prime}} L\right\|_{\infty} \leq\left\|(\Psi, \mathcal{A})_{\left[\frac{N}{2}\right]+1}\right\|_{\infty}$. By $N^{\prime}-2 \geq\left[\frac{N}{2}\right]+1$ and by Lemma $7.1,\left\|(\Psi, \mathcal{A})_{\left[\frac{N}{2}\right]+1}\right\|_{\infty}$ is bounded by $(6.1)$. So our claim holds.

Still assuming $\left|J^{\prime}\right|>\left[\frac{N}{2}\right]$ and $\left|K^{\prime}\right| \leq\left[\frac{N}{2}\right]$, we consider

$$
\begin{aligned}
& \left\|\left(\partial_{T} Z^{J^{\prime}} \Sigma-\frac{1}{T} Z^{J^{\prime}} \Sigma\right)\left(\partial_{T} Z^{K^{\prime}} L-\frac{1}{T} Z^{K^{\prime}} L\right)\right\|_{2} \leq \frac{1}{T}\left\|Z^{J^{\prime}} \Sigma\right\|_{2}\left\|\partial_{T} Z^{K^{\prime}} L\right\|_{\infty} \\
& +\frac{1}{T^{2}}\left\|Z^{J^{\prime}} \Sigma\right\|_{2}\left\|Z^{K^{\prime}} L\right\|_{\infty}+C\left(\mu^{\prime}\right) \epsilon \tilde{E}^{\frac{1}{2}}\left(T, \Sigma_{N}\right),
\end{aligned}
$$

where we expanded the lhs and used Lemma 7.1. For $T \in\left[T_{0}, T^{*}[\right.$ we have

$$
\frac{1}{T}\left\|\Sigma_{N}(T)\right\|_{2} \leq \frac{1}{T}\left\|\Sigma_{N}\left(T_{0}\right)\right\|_{2}+\frac{1}{T} \int_{T_{0}}^{T}\left\|\partial_{T} \Sigma_{N}(\tau)\right\|_{2} d \tau .
$$

Since $\left|K^{\prime}\right| \leq\left[\frac{N}{2}\right]$, by Lemma 7.1

$$
\operatorname{rhs}(1) \leq \frac{C\left(\mu^{\prime}\right) \epsilon}{T}\left(\epsilon+\int_{T_{0}}^{T}\left\|\partial_{T} \Sigma_{N}(\tau)\right\|_{2} d \tau\right)+C\left(\mu^{\prime}\right) \epsilon \tilde{E}^{\frac{1}{2}}\left(T, \Sigma_{N}\right) .
$$

So far we had $k=0$. Now let $k>0$ and set

$$
Z_{3}^{k} Z^{I^{\prime}} \mathcal{Q}_{0}(\Sigma, L)=\left[\frac{1}{T}\left(T \partial_{T}\right)\right]^{k} Z^{I^{\prime}} \mathcal{Q}_{0}(\Sigma, L)
$$

By elementary computation and by Lemma 5.3 this is a sum of terms of the form

$$
\frac{1}{T^{a}} \mathcal{Q}_{0}\left(\left(T \partial_{T}\right)^{c} Z^{J^{\prime}} \Sigma,\left(T \partial_{T}\right)^{d} Z^{K^{\prime}} L\right)
$$

where $\left|J^{\prime}\right|+\left|K^{\prime}\right| \leq\left|I^{\prime}\right|, c+d \leq b \leq k \leq a$. Distinguishing between cases $c+\left|J^{\prime}\right| \leq$ $\left[\frac{N}{2}\right]$ and $c+\left|J^{\prime}\right|>\left[\frac{N}{2}\right]$, and using the fact that the $T$ 's in the numerator are canceled by the $T$ 's in the denominator, we prove the desired estimate proceeding as in the $k=0$ case.

Lemma 7.6. Using the notation of Lemma 7.5 we have for $|I| \leq N^{\prime}$

$$
\left\|Z^{I} \mathcal{Q}_{0}(\Sigma, L)\right\|_{2} \leq \frac{C\left(\mu^{\prime}\right) \epsilon}{T^{\frac{1}{2}-\delta}}\left(\epsilon+\frac{E_{N}^{\frac{1}{2}}(T)}{T}\right) .
$$

We consider $Z_{3}^{k} Z^{I^{\prime}} \mathcal{Q}_{0}(\Sigma, L)$, where $Z^{I^{\prime}}$ does not contain $Z_{3}$. By Lemma 5.3

$$
Z^{I^{\prime}} \mathcal{Q}_{0}(\Sigma, L)=\sum C_{J^{\prime} K^{\prime}} \mathcal{Q}_{0}\left(Z^{J^{\prime}} \Sigma, Z^{K^{\prime}} L\right)
$$

with $C_{J^{\prime} K^{\prime}}$ constants. Suppose $k=0$. We claim:

Claim. Consider

$$
\frac{1}{T^{2}}\left\|\left(\partial_{R} Z^{J^{\prime}} \Sigma\right)\left(\partial_{R} Z^{K^{\prime}} L\right)+\frac{1}{\sinh ^{2}(R)}\left(\partial_{\theta} Z^{J^{\prime}} \Sigma\right)\left(\partial_{\theta} Z^{K^{\prime}} L\right)\right\|_{2} .
$$

Then $(1) \leq \frac{C\left(\mu^{\prime}\right) \epsilon E_{N}^{\frac{1}{2}}(T)}{T^{\frac{3}{2}-\delta}}$ if $\left|I^{\prime}\right| \leq N^{\prime}$.

Proof of the Claim. $\left|I^{\prime}\right| \leq N^{\prime}$ implies $\left|K^{\prime}\right| \leq N^{\prime}$, and by Sobolev inequality

$$
\left\|\partial_{R} Z^{K^{\prime}} L\right\|_{\infty}+\left\|\frac{1}{\sinh (R)} \partial_{\theta} Z^{K^{\prime}} L\right\|_{\infty} \leq C E_{N}^{\frac{1}{2}}(T) .
$$


This and the following inequality, a consequence of $\left|J^{\prime}\right| \leq N^{\prime}$ and (6.1), give us the Claim:

$$
\left\|\partial_{R} Z^{J^{\prime}} \Sigma\right\|_{2}+\left\|\frac{1}{\sinh (R)} \partial_{\theta} Z^{J^{\prime}} \Sigma\right\|_{2} \leq C\left(\mu^{\prime}\right) \epsilon T^{\frac{1}{2}+\delta} .
$$

We now consider

$$
\left\|\left(\partial_{T} Z^{J^{\prime}} \Sigma-\frac{1}{T} Z^{J^{\prime}} \Sigma\right)\left(\partial_{T} Z^{K^{\prime}} L-\frac{1}{T} Z^{K^{\prime}} L\right)\right\|_{2} .
$$

If $\left|K^{\prime}\right|<N^{\prime} / 2$, then by Sobolev embedding and by (6.1),

$$
\begin{aligned}
& (1) \leq \frac{1}{T}\left\|Z^{J^{\prime}} \Sigma\right\|_{2}\left\|\partial_{T} Z^{K^{\prime}} L\right\|_{\infty} \\
& +\frac{1}{T^{2}}\left\|Z^{J^{\prime}} \Sigma\right\|_{2}\left\|Z^{K^{\prime}} L\right\|_{\infty}+\left\|\partial_{T} Z^{J^{\prime}} \Sigma\right\|_{2}\left\|\left(\partial_{T} Z^{K^{\prime}} L-\frac{1}{T} Z^{K^{\prime}} L\right)\right\|_{\infty} \\
& \leq C\left(\mu^{\prime}\right)\left\|L_{N^{\prime}}\right\|_{2}\left(\frac{\left\|Z^{J^{\prime}} \Sigma\right\|_{2}}{T}+\left\|\partial_{T} Z^{J^{\prime}} \Sigma\right\|_{2}\right) \leq C\left(\mu^{\prime}\right) \epsilon^{2} T^{-\frac{1}{2}+\delta},
\end{aligned}
$$

where we used (2) in Lemma 7.5, with $N$ replaced by $N^{\prime}$.

If instead $\left|K^{\prime}\right|>N^{\prime} / 2$, then by (6.1) and by Lemma 7.1 we have

$$
\begin{aligned}
& (1) \leq \frac{1}{T}\left\|Z^{J^{\prime}} \Sigma\right\|_{\infty}\left\|\partial_{T} Z^{K^{\prime}} L\right\|_{2} \\
& +\frac{1}{T^{2}}\left\|Z^{J^{\prime}} \Sigma\right\|_{\infty}\left\|Z^{K^{\prime}} L\right\|_{2}+\left\|\partial_{T} Z^{J^{\prime}} \Sigma\right\|_{\infty}\left\|\left(\partial_{T} Z^{K^{\prime}} L-\frac{1}{T} Z^{K^{\prime}} L\right)\right\|_{2} \\
& \leq C\left(\mu^{\prime}\right) \epsilon\left(\frac{\left\|Z^{J^{\prime}} \Sigma\right\|_{2}}{T}+\left\|\partial_{T} Z^{J^{\prime}} \Sigma\right\|_{2}\right) \leq C\left(\mu^{\prime}\right) \epsilon^{2} T^{-\frac{1}{2}+\delta} .
\end{aligned}
$$

Now let $k>0$ and set

$$
Z_{3}^{k} Z^{I^{\prime}} \mathcal{Q}_{0}(\Sigma, L)=\left[\frac{1}{T}\left(T \partial_{T}\right)\right]^{k} Z^{I^{\prime}} \mathcal{Q}_{0}(\Sigma, L)
$$

By elementary computation and by Lemma 5.3 this is a sum of terms of the form

$$
\frac{1}{T^{a}} \mathcal{Q}_{0}\left(\left(T \partial_{T}\right)^{c} Z^{J^{\prime}} \Sigma,\left(T \partial_{T}\right)^{d} Z^{K^{\prime}} L\right)
$$

where $\left|J^{\prime}\right|+\left|K^{\prime}\right| \leq\left|I^{\prime}\right|, c+d \leq b \leq k \leq a$. Using the fact that the $T^{\prime}$ 's in the numerator are canceled by the $T$ 's in the denominator, we prove the desired estimate proceeding as in the $k=0$ case.

Lemma 7.7. Assume (6.1). Then for $|I| \leq N$ we have

$$
\left\|Z^{I} \mathcal{Q}_{0}(\Sigma, \Sigma)\right\|_{2} \leq \frac{1}{T^{\frac{1}{2}-\delta}}(7.6)
$$

We write as in Lemma 7.5,

$$
Z^{I} \mathcal{Q}_{0}(\Sigma, \Sigma)=\left[\frac{1}{T}\left(T \partial_{T}\right)\right]^{k} Z^{I^{\prime}} \mathcal{Q}_{0}(\Sigma, \Sigma),
$$

where $Z^{I^{\prime}}$ does not contain $\partial_{T}$. The last expression is a sum of terms of the form $\frac{1}{T^{a}}\left(T \partial_{T}\right)^{b} Z^{I^{\prime}} \mathcal{Q}_{0}, b+\left|I^{\prime}\right| \leq|I|$. This in turn is a sum of terms like

$$
\frac{1}{T^{a}} \mathcal{Q}_{0}\left(\left(T \partial_{T}\right)^{c} Z^{J} \Sigma,\left(T \partial_{T}\right)^{d} Z^{K} \Sigma\right)
$$


with $|J|+|K| \leq\left|I^{\prime}\right|$ and $c+d \leq b$. Since one of $c+|J|$ and $b+|K|$ is less than $\left[\frac{N}{2}\right]$ we conclude

$$
\begin{aligned}
& \left\|Z^{I} \mathcal{Q}_{0}(\Sigma, \Sigma)\right\|_{2} \leq\left(\tilde{E}^{\frac{1}{2}}\left(T, \Sigma_{N}\right)+T^{-1}\left\|\Sigma_{N}\right\|_{2}\right) \\
& \left.\times \|\left|\partial_{T} \Sigma_{\left[\frac{N}{2}\right]}\right|+T^{-1}\left|\Sigma_{\left[\frac{N}{2}\right]}\right|+T^{-1}\left|\partial_{R} \Sigma_{\left[\frac{N}{2}\right]}\right|+\frac{\left|\partial_{\theta} \Sigma_{\left[\frac{N}{2}\right]}\right|}{T \sinh (R)}\right)(T) \|_{\infty} .
\end{aligned}
$$

The second factor in the rhs can be bounded by $\frac{C\left(\mu^{\prime}\right) \epsilon}{T^{\frac{1}{2}-\delta}}$ by Lemma 7.1. $T^{-1}\left\|\Sigma_{N}\right\|_{2}$ can be bounded by (2) in Lemma 7.5 .

Lemma 7.8. With the notation of Lemma 7.7, we have for $|I| \leq N^{\prime}$

$$
\left\|Z^{I} \mathcal{Q}_{0}(\Sigma, \Sigma)\right\|_{2} \leq \frac{C\left(\mu^{\prime}\right) \epsilon^{2}}{T^{1-2 \delta}}
$$

Indeed we have an inequality like (1) in Lemma 7.7 with $N^{\prime}$ replacing $N$. Then $\left\|\left(\left|\partial_{T} \Sigma_{\left[\frac{N^{\prime}}{2}\right]}\right|+T^{-1}\left|\Sigma_{\left[\frac{N^{\prime}}{2}\right]}\right|+T^{-1}\left|\partial_{R} \Sigma_{\left[\frac{N^{\prime}}{2}\right]}\right|+\frac{\left|\partial_{\theta} \Sigma_{\left[\frac{N^{\prime}}{2}\right]}\right|}{T \sinh (R)}\right)(T)\right\|_{\infty} \leq C\left(\mu^{\prime}\right) \epsilon T^{-\frac{1}{2}+\delta}$

by Lemma 7.1 . We have

$$
\left(\tilde{E}^{\frac{1}{2}}\left(T, \Sigma_{N^{\prime}}\right)+T^{-1}\left\|\Sigma_{N^{\prime}}\right\|_{2}\right) \leq C\left(\mu^{\prime}\right) \epsilon T^{-\frac{1}{2}+\delta}
$$

by (6.1) and by formula (2) in Lemma 7.5.

By the Leibnitz rule, Lemmas 7.7 and 7.8 and (6.1) imply:

Lemma 7.9. Assume (6.1). Let as in Lemma 7.5, $L=\lambda\langle\Psi(T, x, Y), \psi(x)\rangle_{L_{x}^{2}}+$ $\mu \mathcal{A}(T, Y)$. Then, for $|I| \leq N$ we have

$$
\left\|Z^{I} L \mathcal{Q}_{0}(\Sigma, \Sigma)\right\|_{2} \leq \frac{C\left(\mu^{\prime}\right) \epsilon}{T^{\frac{1}{2}-\delta}}(7.6) .
$$

With the above notation, for $|I| \leq N^{\prime}$ we have

$$
\left\|Z^{I} L \mathcal{Q}_{0}(\Sigma, \Sigma)\right\|_{2} \leq \frac{C\left(\mu^{\prime}\right) \epsilon^{3}}{T^{1-2 \delta}} .
$$

It is enough to bound for $I=J+K$

$$
\left\|\left(Z^{J} L\right) Z^{K} \mathcal{Q}_{0}(\Sigma, \Sigma)\right\|_{2} \text {. }
$$

For $|I| \leq N$ for $|J| \leq N / 2$, by Lemmas 7.1 and 7.7 we have

$$
(1) \leq\left\|Z^{J} L\right\|_{\infty}\left\|Z^{K} \mathcal{Q}_{0}(\Sigma, \Sigma)\right\|_{2} \leq \frac{C\left(\mu^{\prime}\right) \epsilon}{T^{\frac{1}{2}-\delta}}(7.6) .
$$

For $|I| \leq N$ for $|J|>N / 2$, by Sobolev embedding and by Lemma 7.8 we have

$$
(1) \leq\left\|Z^{J} L\right\|_{2} \sum_{|H| \leq N^{\prime}}\left\|Z^{H} \mathcal{Q}_{0}(\Sigma, \Sigma)\right\|_{2} \leq \frac{C\left(\mu^{\prime}\right) \epsilon^{2}}{T^{1-2 \delta}} E_{N}^{\frac{1}{2}}(T) \leq \frac{C\left(\mu^{\prime}\right) \epsilon}{T^{1-2 \delta}}(7.6) .
$$

When $|I| \leq N^{\prime}$ for $|J| \leq N^{\prime} / 2$ by Lemmas 7.1 and 7.8 we have

$$
(1) \leq\left\|Z^{J} L\right\|_{\infty}\left\|Z^{K} \mathcal{Q}_{0}(\Sigma, \Sigma)\right\|_{2} \leq \frac{C\left(\mu^{\prime}\right) \epsilon^{3}}{T^{1-2 \delta}} .
$$

When $|I| \leq N^{\prime}$ for $|J|>N^{\prime} / 2$ we have

$$
(1) \leq\left\|Z^{J} L\right\|_{2} \sum_{|H| \leq N^{\prime}}\left\|Z^{H} \mathcal{Q}_{0}(\Sigma, \Sigma)\right\|_{2} \leq \frac{C\left(\mu^{\prime}\right) \epsilon^{3}}{T^{1-2 \delta}} .
$$


Lemma 7.10. Assume (6.1). Let $|I|+m \leq N$. Set $L=B^{m} \Psi, \mathcal{A}, \Sigma$. Then there is a fixed constant $C$ such that

$$
\left\|\left[Z^{I}, P\right] L\right\|_{2} \leq \frac{C}{T^{2}}\left(E_{N}^{\frac{1}{2}}(T)+\tilde{E}_{N}^{\frac{1}{2}}(T)\right) .
$$

We write $\left[Z^{I}, P\right] L=\left[\partial_{T}^{k}, P\right] Z^{I^{\prime}} L$, where $Z^{I^{\prime}}$ does not contain $\partial_{T}$. This is a sum of terms of the form

$$
\frac{1}{T^{k_{1}+2}} \Delta_{h y p} Z^{\tilde{I}} L, \quad Z^{\tilde{I}}=\partial_{T}^{k_{2}} Z^{I^{\prime}}
$$

with $k_{1}>0, k_{1}+k_{2}=k$ and $|\tilde{I}| \leq|I|-1$. Then apply to the terms in (1) the following lemma:

Lemma 7.11. For $L$ as in Lemma $\% .10$ we have for $|I|+m \leq q-1$ and for a fixed C

$$
\left\|\frac{1}{T} \Delta_{h y p} Z^{I} L\right\|_{2} \leq C\left(E_{q}^{\frac{1}{2}}(T)+\tilde{E}_{q}^{\frac{1}{2}}(T)\right)
$$

where the $L^{2}$ norm is either the $L_{x Y}^{2}$ one for $L=B^{m} \Psi$ or the $L_{Y}^{2}$ one for $L=\mathcal{A}, \Sigma$.

Write $\left\|\frac{1}{T} \Delta_{h y p} Z^{I} L\right\|_{2}=\|\ldots\|_{R \leq 1}+\|\ldots\|_{R \geq 1}$. Then

$$
\left\|\frac{1}{T} \Delta_{h y p} Z^{I} L\right\|_{R \geq 1} \lesssim E_{q}^{\frac{1}{2}}(T)+\tilde{E}_{q}^{\frac{1}{2}}(T)
$$

by $\Delta_{h y p}=\partial_{R}^{2}+\frac{1}{\tanh (R)} \partial_{R}+\frac{1}{\sinh ^{2}(R)} \partial_{\theta}^{2}$. To prove $\left\|\frac{1}{T} \Delta_{h y p} Z^{I} L\right\|_{R \leq 1} \lesssim E_{q}^{\frac{1}{2}}(T)+$ $\tilde{E}_{q}^{\frac{1}{2}}(T)$, by $(5.2)$ we write

$$
\frac{1}{T}\left|\Delta_{h y p} Z^{I} L\right| \leq\left|\frac{Z_{1}}{T} Z_{1} Z^{I} L\right|+\left|\frac{Z_{2}}{T} Z_{2} Z^{I} L\right|+\left|\frac{Z_{0}}{T} Z_{0} Z^{I} L\right|
$$

and see that by the formulas

$$
\begin{aligned}
& Z_{1}=\frac{Y_{1}}{R} \partial_{R}-\frac{1}{\tanh (R)} \frac{Y_{2}}{R} \partial_{\theta} \quad, \quad Z_{0}=\partial_{\theta}, \\
& Z_{2}=\frac{Y_{2}}{R} \partial_{R}+\frac{1}{\tanh (R)} \frac{Y_{1}}{R} \partial_{\theta}
\end{aligned}
$$

we have that $\frac{1}{T}\left\|\Delta_{h y p} Z^{I} L\right\|_{L^{2}(R \leq 1)}$ is bounded by a fixed constant times

$$
\left\|\sum_{j=1}^{2}\left|\frac{\partial_{R}}{T} Z_{j} Z^{I} L\right|+\sum_{j=0}^{2}\left|\frac{\partial_{\theta}}{T \sinh (R)} Z_{j} Z^{I} L\right|\right\|_{2} \leq C\left(E_{q}^{\frac{1}{2}}(T)+\tilde{E}_{q}^{\frac{1}{2}}(T)\right) .
$$

\section{§8. ENERGy INEQUALITIES}

We set

$$
\begin{aligned}
E_{1 \Sigma}\left(T, \Psi_{q}\right)= & E_{(1)}\left(T, \Psi_{q}\right)+\sum_{m+|I| \leq q} \int\left\{2 r_{T x} \partial_{x}\left(B^{m} Z^{I} \Psi\right) \partial_{T}\left(B^{m} Z^{I} \Psi\right)\right. \\
& \left.-\sum_{\alpha, \beta} r_{\alpha \beta} \partial_{\alpha}\left(B^{m} Z^{I} \Psi\right) \partial_{\beta}\left(B^{m} Z^{I} \Psi\right)\right\} \sinh (R) d x d R d \theta
\end{aligned}
$$


with $\alpha$ and $\beta$ summed over all $T, R, x$ and $Y_{j}, j=1,2$, with $r_{\alpha \beta}=0$ if it is not in the list (7.3)-(7.4). We set $E_{\Sigma}\left(T,(\Psi, \mathcal{A})_{q}\right)=E_{1 \Sigma}+E_{(2)}$. For $\epsilon_{0}\left(\mu^{\prime}\right)$ small, (6.1) and Lemma 7.1 imply for any $q \leq N$

$$
\frac{1}{2} E_{q}(T) \leq E_{\Sigma}\left(T, \Psi_{q}\right) \leq 2 E_{q}(T) \quad \forall T \in\left[T_{0}, T^{*}[\quad \forall q \leq N .\right.
$$

Lemma 8.1. There is an $\epsilon\left(\mu^{\prime}\right)>0$ and fixed constants $C_{0}$ and $C_{1}$ such that if (6.1) is true for $\epsilon \in] 0, \epsilon\left(\mu^{\prime}\right)\left[\right.$, then for any $T \in\left[T_{0}, T^{*}[\right.$ we have:

$$
E\left(T,(\Psi, \mathcal{A})_{N}\right)+\tilde{E}\left(T, \Sigma_{N}\right) \leq C_{0} T^{C_{1} C\left(\mu^{\prime}\right) \epsilon} \epsilon^{2} .
$$

Remark. By adjusting $C\left(\mu^{\prime}\right)$ we simply write $E_{N}+\tilde{E}_{N} \leq C_{0} T^{C\left(\mu^{\prime}\right) \epsilon} \epsilon^{2}$.

Proof of Lemma 8.1. Let us set

$$
\begin{aligned}
& \frac{C\left(\mu^{\prime}\right) \epsilon^{2}}{T}+\left(\frac{C\left(\mu^{\prime}\right) \epsilon}{T}+\frac{C}{T^{2}}\right)\left[E_{N}^{\frac{1}{2}}+\tilde{E}_{N}^{\frac{1}{2}}\right](T)+\frac{C\left(\mu^{\prime}\right) \epsilon}{T^{\frac{5}{2}-\delta}} E_{N}(T) \\
& +\frac{C\left(\mu^{\prime}\right) \epsilon}{T^{2}} \int_{T_{0}}^{T}\left[E_{N}^{\frac{1}{2}}+\tilde{E}_{N}^{\frac{1}{2}}\right](\tau) d \tau .
\end{aligned}
$$

Then we have:

Lemma 8.2. Given (6.1) in $\left[T_{0}, T^{*}\left[\right.\right.$ for any $q, q \leq N$, we have $\frac{d}{d T} \tilde{E}^{\frac{1}{2}}\left(T, \Sigma_{q}\right) \leq$ $(8.2)$.

Lemma 8.3. Given (6.1) in $\left[T_{0}, T^{*}\left[\right.\right.$ for any $q, q \leq N$ we have $\frac{d}{d T} E_{\Sigma}^{\frac{1}{2}}\left(T,(\Psi, \mathcal{A})_{q}\right) \leq$ (8.2).

We assume Lemmas 8.2 and 8.3 and continue the proof of Lemma 8.1. By a continuity argument we assume initially that the last term in the first line of (8.2), that is $\frac{C\left(\mu^{\prime}\right) \epsilon}{T^{\frac{5}{2}-\delta}} E_{N}(T)$, is not present. Setting $D=E_{\Sigma}^{\frac{1}{2}}\left(T,(\Psi, \mathcal{A})_{N}\right)+\tilde{E}^{\frac{1}{2}}\left(T, \Sigma_{N}\right)$, $\varphi(T)=2\left(\frac{C\left(\mu^{\prime}\right) \epsilon}{T}+\frac{C}{T^{2}}\right)$, adding from Lemmas 8.2 and 8.3 and using (8.1) we obtain

$$
\frac{d}{d T} D \leq \frac{2 C\left(\mu^{\prime}\right) \epsilon^{2}}{T}+\varphi(T) D+\frac{2 C\left(\mu^{\prime}\right) \epsilon}{T^{2}} \int_{T_{0}}^{T} D(\tau) d \tau
$$

Integrating,

$$
D(T) \leq D\left(T_{0}\right)+2 C\left(\mu^{\prime}\right) \epsilon^{2} \log \frac{T}{T_{0}}+\int_{T_{0}}^{T} \varphi(\tau) D(\tau) d \tau+\int_{T_{0}}^{T} \frac{2 C\left(\mu^{\prime}\right) \epsilon}{\tau} D(\tau) d \tau .
$$

By Gronwall inequality we obtain an inequality of the desired form

$$
D(T) \leq e^{\int_{T_{0}}^{T}\left(\frac{4 C\left(\mu^{\prime}\right) \epsilon}{\tau}+\frac{2 C}{\tau^{2}}\right) d \tau}\left(D\left(T_{0}\right)+2 C\left(\mu^{\prime}\right) \epsilon^{2} \log \frac{T}{T_{0}}\right) .
$$

By a continuity argument it is easy now to absorb the $\frac{C\left(\mu^{\prime}\right) \epsilon}{T^{\frac{5}{2}-\delta}} E_{N}(T)$ term inside $\frac{C}{T^{2}} E_{N}^{\frac{1}{2}}(T)$.

We return now to Lemmas 8.2 and 8.3. Apply $Z^{I}$ to (7.1):

$$
P Z^{I} \Sigma=Z^{I} \frac{\mathcal{H}}{\Omega}+\left[Z^{I}, P\right] \Sigma .
$$


Taking $m \geq 0$ with $B^{0}=P_{c}$, apply $B^{m} Z^{I}$ to $(7.5)$ :

$$
\begin{aligned}
& \left(P+B^{2}\right) B^{m} Z^{I} \Psi+\sum_{\alpha} r_{x \alpha} \partial_{x} \partial_{\alpha} B^{m} Z^{I} \Psi=-B^{m} Z^{I}\left\{\frac{1}{T} F_{2}(\Psi, \mathcal{A})\right. \\
& \left.+\frac{1}{T^{2}} F_{3}(\Psi, \mathcal{A})\right\}+Z^{I}\left[\frac{2}{T} \mathcal{Q}_{0}(\Sigma, \mathcal{A})\right] B^{m} \phi^{\prime}-Z^{I}\left[\frac{1}{T} \mathcal{Q}_{0}(\Sigma, \Sigma)\right] B^{m} \mathrm{th}^{\prime \prime} \\
& -Z^{I}\left[\frac{1}{T^{2}} \mathcal{A} \mathcal{Q}_{0}(\Sigma, \Sigma)\right] B^{m} \phi^{\prime \prime}+Z^{I} B^{m}\left[\frac{1}{T}\left(\Psi_{x}+\mathcal{A} \phi^{\prime}\right) \frac{\mathcal{H}}{\Omega}\right] \\
& -\left[Z^{I}, P\right] B^{m} \Psi-\sum_{\alpha}\left[Z^{I}, r_{x \alpha}\right] B^{m} \Psi_{x \alpha}-\sum_{\alpha} r_{x \alpha}\left[B^{m}, \partial_{x} \partial_{\alpha}\right] Z^{I} \Psi .
\end{aligned}
$$

Finally there is a similar equation for $\mathcal{A}$.

$$
\begin{aligned}
& \left(P+\frac{3}{2}\right) Z^{I} \mathcal{A} \\
& =-Z^{I}\left[\frac{1}{T} G_{2}(\Psi, \mathcal{A})+\frac{1}{T^{2}} G_{3}(\Psi, \mathcal{A})+\frac{1}{T} \mathcal{Q}_{0}(\Sigma, \Sigma)+\frac{1}{T}\left\langle\Psi, \phi^{\prime \prime}\right\rangle \frac{\mathcal{H}}{\Omega}\right] \\
& -Z^{I}\left[\frac{2}{T} \mathcal{Q}_{0}(\Sigma, L(\Psi, \mathcal{A}))+\frac{1}{T^{2}} \mathcal{Q}_{0}(\Sigma, \Sigma) L(\Psi, \mathcal{A})\right]-\left[Z^{I}, P\right] \mathcal{A} .
\end{aligned}
$$

By Lemma 6.1, Lemmas 8.3 and 8.4 follow from:

Lemma 8.4. For $j=3,4,5$ we have $\|r h s(8 . j)\|_{2} \leq(8.2)$.

Lemmas 7.2 and 7.4 guarantee, for $m+|I| \leq N$, that for $\ell=2,3$, quadratic or cubic expressions succinctly denoted by $(\Psi, \mathcal{A})^{\ell}$ satisfy

$$
\left\|B^{m} Z^{I} \frac{1}{T^{\ell-1}}(\Psi, \mathcal{A})^{\ell}\right\|_{2} \leq \frac{C\left(\mu^{\prime}\right)}{T^{\ell-1}} \epsilon E_{N}^{\frac{1}{2}}(T) .
$$

Lemma 7.3 guarantees for $|I| \leq N^{\prime}$

$$
\left\|Z^{I} \frac{1}{T^{\ell-1}}(\langle\Psi, \psi\rangle, \mathcal{A})^{\ell}\right\|_{2} \leq \frac{C\left(\mu^{\prime}\right)}{T^{\ell-1}} \epsilon^{\ell} .
$$

Lemma 7.5 guarantees for $|I| \leq N$

$$
\left\|Z^{I} \frac{1}{T} \mathcal{Q}_{0}(\Sigma, L(\langle\Psi, \psi\rangle, \mathcal{A}))\right\|_{2} \leq \frac{(7.6)}{T} \leq(8.2)
$$

and Lemma 7.6 guarantees for $|I| \leq N^{\prime}$

$$
\begin{aligned}
& \left\|Z^{I} \frac{1}{T} \mathcal{Q}_{0}(\Sigma, L(\langle\Psi, \psi\rangle, \mathcal{A}))\right\|_{2} \leq \frac{C\left(\mu^{\prime}\right) \epsilon}{T^{\frac{3}{2}}-\delta}\left(\epsilon+\frac{E_{N}^{\frac{1}{2}}(T)}{T}\right) \text { for }|I|=N^{\prime}, \\
& \left\|Z^{I} \frac{1}{T} \mathcal{Q}_{0}(\Sigma, L(\langle\Psi, \psi\rangle, \mathcal{A}))\right\|_{2} \leq \frac{C\left(\mu^{\prime}\right) \epsilon^{2}}{T^{\frac{3}{2}-\delta}} \text { for }|I|<N^{\prime} .
\end{aligned}
$$

Lemma 7.7 guarantees for $|I| \leq N$

$$
\left\|Z^{I} \frac{1}{T} \mathcal{Q}_{0}(\Sigma, \Sigma)\right\|_{2} \leq \frac{(7.6)}{T^{\frac{3}{2}-\delta}} \leq(8.2)
$$

and Lemma 7.8 guarantees for $|I| \leq N^{\prime}$

$$
\left\|Z^{I} \frac{1}{T} \mathcal{Q}_{0}(\Sigma, \Sigma)\right\|_{2} \leq \frac{C\left(\mu^{\prime}\right) \epsilon^{2}}{T^{2-2 \delta}} .
$$


Lemma 7.9 guarantees for $|I| \leq N$

$$
\left\|Z^{I} \frac{1}{T^{2}} L(\langle\Psi, \psi\rangle, \mathcal{A}) \mathcal{Q}_{0}(\Sigma, \Sigma)\right\|_{2} \leq \frac{(7.6)}{T^{\frac{5}{2}-\delta}} \leq(8.2)
$$

and for $|I| \leq N^{\prime}$

$$
\left\|Z^{I} \frac{1}{T^{2}} L(\langle\Psi, \psi\rangle, \mathcal{A}) \mathcal{Q}_{0}(\Sigma, \Sigma)\right\|_{2} \leq \frac{C\left(\mu^{\prime}\right) \epsilon^{3}}{T^{3-2 \delta}} .
$$

The following lemma holds:

Lemma 8.5. We have

$$
\begin{aligned}
& \left\|(\mathcal{H})_{N}\right\|_{2} \leq(8.2), \quad\left\|(\mathcal{H})_{N^{\prime}}\right\|_{2} \leq \frac{C\left(\mu^{\prime}\right) \epsilon}{T^{\frac{3}{2}}-2 \delta}\left(\frac{E_{N}^{\frac{1}{2}}(T)}{T}+\epsilon\right) ; \\
& \left\|(\mathcal{H} / \Omega)_{N}\right\|_{2} \leq(8.2), \quad\left\|(\mathcal{H} / \Omega)_{N^{\prime}}\right\|_{2} \leq \frac{C\left(\mu^{\prime}\right) \epsilon}{T^{\frac{3}{2}}-2 \delta}\left(\frac{E_{N}^{\frac{1}{2}}(T)}{T}+\epsilon\right) .
\end{aligned}
$$

We have

$$
\left\|\left(\frac{\mathcal{H}-\frac{6}{T} \mathcal{A}\left\langle\Psi, t h t h^{\prime} \phi\right\rangle}{\Omega}\right)_{N^{\prime}}\right\|_{2} \leq \frac{C\left(\mu^{\prime}\right) \epsilon}{T^{\frac{3}{2}-\delta}}\left(\frac{E_{N}^{\frac{1}{2}}(T)}{T^{1-\delta}}+\epsilon\right) .
$$

We have

$$
\left\|\left(\operatorname{rhs}(8.5)+Z^{I} \frac{3}{T} \mathcal{A}^{2}\left\|t h \phi^{3}\right\|_{1}+\left[Z^{I}, P\right] \mathcal{A}\right)_{N^{\prime}}\right\|_{2} \leq \frac{C\left(\mu^{\prime}\right) \epsilon}{T^{\frac{3}{2}-2 \delta}}\left(\frac{E_{N}^{\frac{1}{2}}(T)}{T}+\epsilon\right) .
$$

(8.6) follows from inequalities from (1) to (8) in Lemma 8.4 and from Lemma 7.3. (8.7) follows from (8.6) and the Leibnitz rule. (8.9) follows from inequalities from (1) to (8) in Lemma 8.4, Lemma 7.3 and (8.7). (8.8) follows from (1) to (8) in Lemma 8.4, from Lemma 7.3 and the Leibnitz rule because we have eliminated the only term which decays like $T^{-\frac{3}{2}+2 \delta}$, the $\mathcal{A}\left\langle\Psi\right.$, $\left.\operatorname{thth}^{\prime} \phi\right\rangle$ one.

We resume the proof of Lemma 8.4. Lemma 7.10 guarantees for $m+|I| \leq N$

$$
\left\|\left[Z^{I}, P\right]\left(B^{m} \Psi, \mathcal{A}, \Sigma\right)\right\|_{2} \leq \frac{C}{T^{2}}\left(E_{N}^{\frac{1}{2}}+\tilde{E}_{N}^{\frac{1}{2}}\right) \leq(8.2) .
$$

Hence (9) and (8.7) guarantee Lemma 8.4 for $j=3$. (8.7) and the Leibnitz rule guarantee for $|I| \leq N$

$$
\left\|Z^{I}\left(\frac{1}{T}\langle\Psi, \psi\rangle \frac{\mathcal{H}}{\Omega}\right)\right\| \leq(8.2) .
$$

The above estimates guarantee Lemma 8.4 for $j=5$.

Given a linear combination $L=\lambda \Psi(x, Y, T)+\mu \mathcal{A}(Y, T) \psi(x)$, we want to show that for $|I|+m \leq N$ we have

$$
\left\|Z^{I} B^{m} \partial_{x} L \frac{\mathcal{H}}{T \Omega}\right\|_{2} \leq(8.2) .
$$

We consider for $|J|+|K| \leq|I|$

$$
\left\|\left(Z^{J} B^{m} \partial_{x} L\right)\left(Z^{K} \frac{\mathcal{H}}{T \Omega}\right)\right\|_{2} .
$$


If $|K| \leq[N] / 2$, then the desired inequality follows from

$$
\begin{aligned}
& (12) \leq\left\|Z^{J} B^{m} \partial_{x} L\right\|_{2}\left\|Z^{K} \frac{\mathcal{H}}{T \Omega}\right\|_{\infty} \leq C T^{-1} E_{N}^{\frac{1}{2}}(T)\left\|(\mathcal{H} / \Omega)_{N^{\prime}}\right\|_{2} \\
& \leq \frac{C\left(\mu^{\prime}\right) \epsilon}{T^{\frac{5}{2}-2 \delta}} E_{N}^{\frac{1}{2}}(T)\left(\frac{E_{N}^{\frac{1}{2}}(T)}{T}+\epsilon\right) \leq(8.2) .
\end{aligned}
$$

If $|K| \geq[N] / 2+1$, then $|J|+m \leq[N / 2]-2$. Then

$$
(12) \leq\left\|Z^{J} B^{m} \partial_{x} L\right\|_{\infty}\left\|Z^{K} \frac{\mathcal{H}}{T \Omega}\right\|_{2} \leq C\left\|Z^{K} \frac{\mathcal{H}}{T \Omega}\right\|_{2} \sup _{|J|+m \leq N^{\prime}-4}\left\|Z^{J} \partial_{x}^{m} L\right\|_{2} .
$$

Then $\left\|Z^{K} \frac{\mathcal{H}}{T \Omega}\right\|_{2} \leq T^{-1}(8.2)$ by $(8.7)$ and $\left\|Z^{J} \partial_{x}^{m} L\right\|_{2} \leq C T^{\frac{1}{2}-\frac{1}{p}}\left\|Z^{J} \partial_{x}^{m} L\right\|_{L_{y}^{2} L_{x}^{p}}$ by a lemma stated and proved immediately below; see Lemma 8.6. These last two estimates give $(12) \leq(8.2)$ also for $|K| \geq N^{\prime}$. We state and prove Lemma 8.6 and then we continue the proof of Lemma 8.4.

Lemma 8.6. Let $1 \leq p \leq q \leq \infty$ and fix any $j$ and $J$. Assume (6.1). Then there are constants $C$ and $C_{M}$ such that for any $T \in\left[T_{0}, T^{*}[\right.$ and any $Y$ we have for our solution: $\left\|\partial_{x}^{j} Z^{J} \Psi(T, Y)\right\|_{L_{x}^{p}} \leq$

$$
C T^{\frac{1}{p}-\frac{1}{q}}\left(\left\|\partial_{x}^{j} Z^{J} \Psi(T, Y)\right\|_{L_{x}^{q}}+\frac{C_{M}}{T^{M}}\left(\left|Z^{J} \Sigma(T, Y)\right|+\left|Z^{J} \mathcal{A}(T, Y)\right|\right)\right) .
$$

For simplicity let us pick $j+|J|=0$, but the general argument is the same. First of all we write $\int_{\mathbb{R}} d x|\Psi(T, x, Y)|^{p}=\int_{|x+\sigma(t, y)| \leq T}+\int_{|x+\sigma(t, y)| \geq T}$. Then, by Hölder,

$$
\int_{|x+\sigma(t, y)| \leq T} d x|\Psi(T, x, Y)|^{p} \leq T^{1-\frac{p}{q}}\|\Psi(T, x, Y)\|_{L_{x}^{q}}^{p} .
$$

Next we consider the $|x+\sigma(t, y)| \geq T$ integral. In intervals of existence, the solution $w$ of (1.4) is supported in the set $x^{2}+y^{2}=x^{2}+T^{2} \sinh ^{2} R \leq(t+K)^{2}<(t+2 K)^{2}=$ $T^{2} \cosh ^{2} R$. This implies that $T \geq|x|$ on the support of $w$. By (1.10) and (3.2)

$\psi(t, x, y)=w(t, x+\sigma(t, y), y)+\sigma(t, y) \int_{0}^{1} \operatorname{th}^{\prime}(x+\sigma(t, y)-s \sigma(t, y)) d s-\phi(x) a(t, y)$.

So, for $|x+\sigma(t, y)| \geq T$,

$$
\psi(t, x, y)=\sigma(t, y) \int_{0}^{1} \operatorname{th}^{\prime}(x+s \sigma(t, y)) d s-\phi(x) a(t, y) .
$$

Lemma 7.1 and the equality $\sigma=\frac{\Sigma}{T}$ imply (here we are focusing on the $\sigma$ 's in the argument of $w$ and $t^{\prime}$ )

$$
|\sigma(t, y)| \leq C \mu^{\prime} T^{-\frac{1}{2}+\delta} \epsilon .
$$

Therefore, for $\epsilon$ small, inequality $|x+\sigma(t, y)| \geq T$ implies $|x|>T / 2$. Therefore, for some fixed constants $C$ and $C_{M}$,

$$
\begin{aligned}
& \int_{|x+\sigma(t, y)| \geq T} d x|\Psi(T, x, Y)|^{p} \\
& \leq C|\Sigma(T, Y)|^{p} \int_{|x| \geq T / 2} d x\left|\operatorname{th}^{\prime}(x)\right|^{p}+C|\mathcal{A}(T, Y)|^{p} \int_{|x| \geq T / 2} d x|\phi(x)|^{p} \\
& \leq C_{M} T^{-M}\left(|\Sigma(T, Y)|^{p}+|\mathcal{A}(T, Y)|^{p}\right),
\end{aligned}
$$

with $M>0$ an arbitrarily large number. 
To complete the proof of Lemma 8.4 we still need to prove $\|r h s(8.4)\|_{2} \leq(8.2)$. We have $\left\|B^{m} Z^{I} \frac{1}{T} F_{2}(\Psi, \mathcal{A})\right\|_{2} \leq(8.2)$ by $(1),\left\|B^{m} Z^{I} \frac{1}{T^{2}} F_{3}(\Psi, \mathcal{A})\right\|_{2} \leq T^{-1}(8.2)$ by (1), $\left\|B^{m} Z^{I} \frac{1}{T} \mathcal{Q}_{0}(\Sigma, \mathcal{A}) \phi^{\prime}\right\|_{2} \leq(8.2)$ by $(3),\left\|B^{m} Z^{I} \frac{1}{T} \mathcal{Q}_{0}(\Sigma, \Sigma) \operatorname{th}^{\prime \prime}\right\|_{2} \leq$ (8.2) by (5), $\left\|B^{m} Z^{I} \frac{1}{T^{2}} \mathcal{A} \mathcal{Q}_{0}(\Sigma, \Sigma) \phi^{\prime \prime}\right\|_{2} \leq T^{-\frac{3}{2}+\delta}(8.2)$ by $(7),\left\|\left[Z^{I}, P\right] B^{m} \Psi\right\|_{2} \leq$ (8.2) by (9) and $\| B^{m} Z^{I}\left[\frac{1}{T}\left(\Psi_{x}+\mathcal{A} \phi^{\prime}\right) \frac{\mathcal{H}}{\Omega} \|_{2} \leq(8.2)\right.$ by (12). Hence we have $\|r h s(8.4)\|_{2} \leq(8.2)$ if we prove:

Lemma 8.7. Assume (6.1). Then we have for $|I|+m \leq N$ :

$$
\begin{aligned}
& \left\|\left[Z^{I}, r_{x \alpha}\right] B^{m} \Psi_{x \alpha}\right\|_{2} \leq(8.2), \\
& \left\|r_{x \alpha}\left[\partial_{x} \partial_{\alpha}, B^{m}\right] Z^{I} \Psi\right\|_{2} \leq(8.2) .
\end{aligned}
$$

Recall

$$
\begin{aligned}
& r_{x x}=\frac{1}{T^{2}} \mathcal{Q}_{0}(\Sigma, \Sigma) \quad, \quad r_{x R}=r_{R x}=\frac{2}{T^{3}} \Sigma_{R} \\
& r_{x T}=r_{T x}=-\frac{2}{T}\left(\Sigma_{T}-\frac{\Sigma}{T}\right) \quad, r_{x Y_{j}}=r_{Y_{j} x}=\frac{2 \Sigma_{\theta}}{T^{3} \sinh (R)} a_{j}(Y)
\end{aligned}
$$

where $a_{1}(Y)=-\frac{Y_{2}}{\sinh (R)}$ and $a_{2}(Y)=\frac{Y_{1}}{\sinh (R)}$. We prove (1) first. $\left[Z^{I}, r_{x \alpha}\right] B^{m} \Psi_{x \alpha}$ is a sum of terms $\left(Z^{J} B^{m} \Psi_{x \alpha}\right)\left(Z^{K} r_{x \alpha}\right)$, with $m+|J|+|K| \leq|I|$. If $m+|J| \leq\left[\frac{N}{2}\right]$, by Lemma 7.1, $N^{\prime} \geq\left[\frac{N}{2}\right]+3$, Lemma 8.6 and (6.1) we have

$$
\left\|Z^{J} B^{m} \Psi_{x \alpha}\right\|_{L_{Y}^{\infty} L_{x}^{p}} \leq C\left(\mu^{\prime}\right)\left(T^{\frac{1}{2}-\frac{1}{p}}\left\|Z^{J} B^{m} \Psi_{x \alpha}\right\|_{L_{Y}^{\infty} L_{x}^{2}}+\frac{\epsilon}{T}\right) \leq C\left(\mu^{\prime}\right) \epsilon T^{-\frac{1}{p}+2 \delta} .
$$

We have $\left\|Z^{K} r_{x x}\right\|_{2} \leq T^{-1}(8.2)$ by (5) in Lemma 8.4, $C\left(\mu^{\prime}\right) \epsilon\left\|Z^{K} r_{x \alpha}\right\|_{2} \leq T^{-1}(8.2)$ for $\alpha=R, Y_{j}$ by the definitions, and $C\left(\mu^{\prime}\right) \epsilon\left\|Z^{K} r_{x T}\right\|_{2} \leq T^{-1}(8.2)$ which follows by $\left\|Z^{K} \Sigma_{T} / T\right\|_{2} \leq T^{-1} \tilde{E}_{N}^{\frac{1}{2}}(T)$ and by formula (2) in Lemma 7.5.

If $m+|J|>\left[\frac{N}{2}\right]$, then $\left\|Z^{K} r_{x x}\right\|_{2} \leq C\left(\mu^{\prime}\right) T^{-3+2 \delta}$ by (6) in Lemma 8.4. $\left\|Z^{K} r_{x \alpha}\right\|_{2}$ $\leq \mu^{\prime} T^{-2} \epsilon$ for $\alpha \neq x$ by (6.1). We have $\left\|Z^{J} B^{m} \Psi_{x \alpha}\right\|_{2} \leq C E^{\frac{1}{2}}\left(T, \Psi_{N}\right)$, where we use $|J|+m \leq N-1$ and $\left\|Z^{J} B^{m} \Psi_{x \alpha}\right\|_{2} \leq C\left\|Z^{J} B^{m+1} \Psi_{\alpha}\right\|_{2}$ by $\Psi=P_{c}(H) \Psi$. All these estimates imply claim (1) of Lemma 8.7.

We prove (2) in Lemma 8.7. For $\alpha=T, R, Y_{j}$, since $\left[\partial_{x}, B^{m}\right]$ is a pdo of order $m$, since $B$ is elliptic and $\Psi=P_{c}(H) \Psi$

$$
\left\|r_{x \alpha}\left[\partial_{x}, B^{m}\right] \partial_{\alpha} Z^{I} \Psi\right\|_{2} \leq C\left\|r_{x \alpha}\right\|_{\infty}\left\|B^{m} \partial_{\alpha} Z^{I} \Psi\right\|_{2} \leq \frac{C\left(\mu^{\prime}\right) \epsilon}{T^{\frac{3}{2}-\delta}} E_{N}^{\frac{1}{2}}
$$

For $\alpha=x$, since $\left[\partial_{x}^{2}, B^{m}\right]$ is a pdo of order $m+1$,

$$
\left\|r_{x x}\left[\partial_{x}^{2}, B^{m}\right] Z^{I} \Psi\right\|_{2} \leq C\left\|r_{x x}\right\|_{\infty}\left\|B^{m+1} Z^{I} \Psi\right\|_{2} \leq \frac{C\left(\mu^{\prime}\right) \epsilon^{2}}{T^{3-2 \delta}} E_{N}^{\frac{1}{2}} .
$$

\section{$\S 9$. The Elimination of the $Y$ VARIABle}

Following Klainerman (see $[\mathrm{K}],[\mathrm{DFX}]$ ) the energy estimates in the previous section are now used to interpret terms in the equations for $\Psi$ and $\mathcal{A}$, that is (7.2) and (7.3), with derivatives in $Y$, as perturbations integrable in $T$. Hence the equation for $\Psi$ is interpreted as a Schrödinger equation with time $T$ and space variable $x$, 
and the equation for $\mathcal{A}$ is interpreted as an ODE with time $T$. Specifically, we write

$$
\Psi_{T T}+B^{2} \Psi+r_{x T} P_{c} \Psi_{T x}=-\frac{1}{T} P_{c} F_{2}(\Psi, \mathcal{A})-\frac{1}{T^{2}} R_{\Psi}
$$

with

$$
\begin{aligned}
R_{\Psi} & =P_{c} F_{3}(\Psi, \mathcal{A})+\Delta_{h y p} \Psi+T \mathcal{Q}_{0}(\Sigma, \Sigma) P_{c} \mathrm{th}^{\prime \prime} \\
& -2 T \mathcal{Q}_{0}(\Sigma, \mathcal{A}) P_{c} \phi^{\prime}+\mathcal{A} \mathcal{Q}_{0}(\Sigma, \Sigma) P_{c} \phi^{\prime \prime} \\
& +Q_{0}(\Sigma, \Sigma) P_{c} \Psi_{x x}+T P_{c}\left(\Psi_{x}+\mathcal{A} \phi^{\prime}\right) P \Sigma-T^{2} \sum_{\alpha \neq T} r_{x \alpha} \Psi_{x \alpha}
\end{aligned}
$$

and we write

$$
\mathcal{A}_{T T}+\frac{3}{2} \mathcal{A}=-\frac{3}{T} \mathcal{A}^{2}\left\|\operatorname{th} \phi^{3}\right\|_{1}-\frac{1}{T^{2}} R_{\mathcal{A}}
$$

with

$$
\begin{aligned}
R_{\mathcal{A}} & =T\left\langle 3 \operatorname{th} \Psi^{2}+6 \operatorname{th} \mathcal{A} \phi \Psi, \phi\right\rangle+G_{3}(\Psi, \mathcal{A})+\mathcal{Q}_{0}(\Sigma, \Sigma)\left\langle\Psi, \phi^{\prime \prime}\right\rangle+T\left\langle\Psi, \phi^{\prime \prime}\right\rangle P \Sigma \\
& +2 T \mathcal{Q}_{0}\left(\Sigma,\left\langle\Psi, \phi^{\prime}\right\rangle\right)+\mathcal{A} Q_{0}(\Sigma, \Sigma)\left\|\phi^{\prime}\right\|_{2}^{2}+\Delta_{h y p} \mathcal{A}+T \mathcal{Q}_{0}(\Sigma, \Sigma)\left\|\operatorname{th}^{\prime \prime} \phi\right\|_{1} .
\end{aligned}
$$

We have:

Lemma 9.1. There are a fixed constant $C$ and an $\epsilon_{0}>0$ such that for $|I| \leq N^{\prime}$ and for $0<\epsilon<\epsilon_{0}$ we have for all $T \in\left[T_{0}, T^{*}[\right.$ :

$$
\left\|Z^{I} R_{\mathcal{A}}(T)\right\|_{L_{Y}^{2}} \leq C T^{\frac{3}{4}} \epsilon .
$$

Lemma 9.1 is a consequence of (8.9) and, by (5.2) and Lemma 8.1, for $L=\mathcal{A}, \Psi$ and for $|I| \leq N-2$, of

$$
\left\|Z^{I} \Delta_{h y p} L\right\|_{2} \leq\left\|L_{|I|+2}\right\|_{2} \leq\left\|L_{N}\right\|_{2} \leq C T^{C\left(\mu^{\prime}\right) \epsilon} \epsilon .
$$

The following two lemmas are proved in $\S 14$.

Lemma 9.2. There are a fixed constant $C$ and an $\epsilon_{0}>0$ such that for $0<\epsilon<\epsilon_{0}$ and for $|I|+m \leq N^{\prime}$ we have for all $T \in\left[T_{0}, T^{*}[\right.$

$$
\left\|Z^{I} B^{m+1} R_{\Psi}\right\|_{L_{x Y}^{2}} \leq C T^{\frac{3}{4}} \epsilon .
$$

Lemma 9.3. There are a fixed constant $C$ and an $\epsilon_{0}>0$ such that for $0<\epsilon<\epsilon_{0}$ and for $|I|+m \leq N^{\prime}$ we have for all $T \in\left[T_{0}, T^{*}[\right.$ :

$$
\left\|B^{m} Z^{I} R_{\Psi}(T)\right\|_{L_{Y}^{2} W_{x}^{1, \frac{p}{p-1}}} \leq C T^{\frac{3}{4}} \epsilon .
$$

\section{§10. Normal Form argument For $\mathcal{A}$}

The starting points are (9.2) and Lemma 9.2. Consider $\mathcal{A}_{ \pm}$related to $\mathcal{A}$ by

$$
\mathcal{A}_{ \pm}=\left(\mp i \partial_{T}+\sqrt{3 / 2}\right) \mathcal{A} \quad, \quad \mathcal{A}=\frac{\mathcal{A}_{+}+\mathcal{A}_{-}}{\sqrt{6}} .
$$

Then write

$$
\left( \pm i \partial_{T}+\sqrt{3 / 2}\right) \mathcal{A}_{ \pm}=-3 \frac{\left\|\operatorname{th} \phi^{3}\right\|_{1}}{6 T}\left(\mathcal{A}_{+}+\mathcal{A}_{-}\right)^{2}-\frac{1}{T^{2}} R_{\mathcal{A}} .
$$


By the theory of normal forms there are constants $\alpha_{ \pm}, \beta_{ \pm}, \gamma_{ \pm}$so that, if we write $\mathcal{A}_{ \pm}=A_{1+}+\frac{\alpha_{ \pm}}{T}\left(A_{1 \pm}\right)^{2}+\frac{\beta_{ \pm}}{T} A_{1 \pm} A_{1 \mp}+\frac{\gamma_{ \pm}}{T}\left(A_{1 \mp}\right)^{2}$, we obtain

$$
\left( \pm i \partial_{T}+\sqrt{3 / 2}\right) A_{1, \pm}=-\frac{1}{T^{2}} R_{\mathcal{A} \pm},
$$

where we have, by Lemma 9.1:

Lemma 10.1. There is a fixed constant $C$ such that for $R_{\mathcal{A}}$ as above we have for all $T \in\left[T_{0}, T^{*}[:\right.$

$$
\sum_{|I| \leq N^{\prime}}\left\|Z^{I} R_{\mathcal{A} \pm}(T)\right\|_{L_{Y}^{2}} \leq C T^{\frac{3}{4}} \epsilon
$$

We have $R_{\mathcal{A}+}-R_{\mathcal{A}}=O\left(A_{1, \pm}^{3}\right)+O\left(T^{-1} A_{1, \pm} R_{\mathcal{A}}\right)$ plus smaller terms. By the Leibnitz rule, Sobolev inequality, (6.1), Lemma 8.1 and $\epsilon$ small $\left\|Z^{I}\left(A_{1,+}, A_{1,-}\right)^{3}\right\|_{2} \leq$

$$
\left\|\left(A_{1,+}, A_{1,-}\right)_{N}\right\|_{2}^{2}\left\|\left(A_{1,+}, A_{1,-}\right)_{N^{\prime}}\right\|_{2} \approx\left\|\left(\mathcal{A}_{+}, \mathcal{A}_{-}\right)_{N}\right\|_{2}^{2}\left\|\left(\mathcal{A}_{+}, \mathcal{A}_{-}\right)_{N^{\prime}}\right\|_{2} \leq C T^{C\left(\mu^{\prime}\right) \epsilon} \epsilon .
$$

We have by (6.1), Lemma 9.1 and $\epsilon$ small

$$
\left\|Z^{I} T^{-1}\left(R_{\mathcal{A}} A_{1, \pm}\right)\right\|_{2} \lesssim T^{-1}\left\|\left(\mathcal{A}_{ \pm}\right)_{N^{\prime}}\right\|_{2}\left\|\left(R_{\mathcal{A}}\right)_{N^{\prime}}\right\|_{2} \leq C \epsilon T^{-\frac{1}{4}}
$$

We have:

Lemma 10.2. Assume (6.1). Then there is a fixed constant $C$ such that $\forall I$ with $|I| \leq N^{\prime}$ we have for all $T \in\left[T_{0}, T^{*}[\right.$

$$
\left\|Z^{I} A_{1 \pm}(T)\right\|_{L_{Y}^{2}} \leq C \epsilon .
$$

For any multiindex $I$ with $|I| \leq N^{\prime}$ we have

$$
\left( \pm i \partial_{T}+\sqrt{3 / 2}\right) Z^{I} A_{1 \pm}=\frac{1}{T} F_{ \pm}^{I} \quad \text { with } \quad\left\|F_{ \pm}^{I}(T)\right\|_{L_{Y}^{2}}+\left\|F_{ \pm}^{I}(T)\right\|_{L_{Y}^{\infty}} \leq C \epsilon T^{-\frac{1}{4}}
$$

Next, we can write

$$
\frac{1}{2} \partial_{T}\left|Z^{I} A_{1 \pm}\right|^{2} \leq \frac{1}{T} \Im\left\{F_{ \pm}^{I} Z^{I} \bar{A}_{1 \pm}\right\}
$$

from which we obtain

$$
\frac{d}{d T}\left\|Z^{I} A_{1 \pm}\right\|_{L_{Y}^{2}} \leq \frac{1}{T}\left\|F_{ \pm}^{I}(T)\right\|_{L_{Y}^{2}} \leq C \epsilon T^{-\frac{5}{4}} .
$$

\section{$\S 11$. BOUNDS ON $\Psi$}

We have:

Lemma 11.1. Assume (6.1) and Lemmas 9.2 and 9.3. Then there are a fixed $c$ and an $\epsilon_{0}\left(\mu^{\prime}\right)$ such that if $\left.\epsilon \in\right] 0, \epsilon_{0}\left(\mu^{\prime}\right)\left[\right.$ and $T \in\left[T_{0}, T^{*}[\right.$ and for any choice of multiindex $I$ and nonnegative integer $m$ with $|I|+m \leq N^{\prime}$

$$
\left\|B^{m} Z^{I} \Psi(T)\right\|_{L_{Y}^{2} L_{x}^{p}} \leq c T^{2 \delta-\frac{1}{2}} \epsilon .
$$

We start from (9.1). Apply $B^{m} Z^{I}$ and consider

$$
\left(B^{m} Z^{I} \Psi\right)_{T T}+B^{2} B^{m} Z^{I} \Psi=-Z^{I} \frac{B^{m}}{T} F_{2}(\Psi, \mathcal{A})-Z^{I} \frac{B^{m}}{T^{2}} R_{\Psi}-Z^{I} r_{x T} B^{m} \Psi_{x T}
$$


We write $B^{m} Z^{I} \Psi(T)=\sum_{j=1}^{4} B^{m} \Psi_{I j}(T)$ where

$$
\begin{aligned}
& B^{m} \Psi_{I 1}(T)=\frac{\sin \left(B\left(T-T_{0}\right)\right)}{B} B^{m} Z^{I} \Psi_{T}\left(T_{0}\right)+\cos \left(B\left(T-T_{0}\right)\right) Z^{I} B^{m} \Psi\left(T_{0}\right), \\
& B^{m} \Psi_{I j}(T)=-\int_{T_{0}}^{T} \frac{\sin B(T-\tau)}{B} \mathcal{F}_{j}(\tau) d \tau, \quad j=2,3,4, \\
& \mathcal{F}_{2}(T)=P_{c} Z^{I} \frac{1}{T} B^{m} F_{2}(\mathcal{A}, \Psi), \quad \mathcal{F}_{3}(T)=Z^{I} \frac{1}{T^{2}} B^{m} R_{\Psi}(T), \\
& \mathcal{F}_{4}(T)=Z^{I} r_{x T} B^{m} \Psi_{T x}(T) .
\end{aligned}
$$

Then we have the following lemma:

Lemma 11.2. We have:

$$
\begin{aligned}
&\left\|B^{m} \Psi_{I 1}(T)\right\|_{L_{Y}^{2} L_{x}^{p}} \leq c\langle T\rangle^{-\frac{1}{2}+\frac{1}{p}} \epsilon, \\
&\left\|B^{m} \Psi_{I 2}(T)\right\|_{L_{Y}^{2} L_{x}^{p}} \leq C\left(\mu^{\prime}\right)\langle T\rangle^{-\frac{1}{2}+\frac{1}{p}} \log (\langle T\rangle) \epsilon^{2}, \\
&\left\|B^{m} \Psi_{I 3}(T)\right\|_{L_{Y}^{2} L_{x}^{p}} \leq C\left(\mu^{\prime}\right)\langle T\rangle^{-\frac{1}{2}+\frac{1}{p}} \epsilon, \\
&\left\|B^{m} \Psi_{I 4}(T)\right\|_{L_{Y}^{2} L_{x}^{p}} \leq C\left(\mu^{\prime}\right)\langle T\rangle^{-\frac{1}{2}+2 \delta} \epsilon^{2} .
\end{aligned}
$$

(1) follows from Proposition 4.1 and Corollary 2.3. (3) Follows from

$$
\begin{aligned}
& \int_{T_{0}}^{T}\left\|\frac{\sin B(T-\tau)}{B} B^{m} Z^{I} R_{\Psi}(\tau)\right\|_{L_{Y}^{2} L_{x}^{p}} \frac{d \tau}{\tau^{2}} \\
& \leq \int_{T_{0}}^{T}\left\|B^{m} Z^{I} R_{\Psi}(\tau)\right\|_{L_{Y}^{2} W_{x}^{1, \frac{p}{p-1}}} \frac{C d \tau}{(T-\tau)^{\frac{1}{2}-\frac{1}{p}} \tau^{2}} \leq \int_{T_{0}}^{T} \frac{C_{1} \epsilon d \tau}{(T-\tau)^{\frac{1}{2}-\frac{1}{p}}} \tau^{-\frac{10}{9}}
\end{aligned}
$$

by Lemma 9.3. As for inequality (2), notice that if we consider

$$
\int_{T_{0}}^{T}\left\|\frac{\sin B(T-\tau)}{B} P_{c} Z^{I} \frac{1}{\tau} B^{m} F_{2}(\mathcal{A} \phi, \Psi)\right\|_{L_{Y}^{2} L_{x}^{p}} d \tau,
$$

then by Corollary 2.3,

$$
(5) \leq c \int_{T_{0}}^{T}(T-\tau)^{-\frac{1}{2}+\frac{1}{p}}\left\|P_{c} Z^{I} \frac{1}{\tau} B^{m} F_{2}(\mathcal{A} \phi, \Psi)\right\|_{L_{Y}^{2} W_{x}^{1, \frac{p}{p-1}}} d \tau .
$$

Now $\tau Z^{I} \frac{1}{\tau} B^{m} F_{2}$ is formed by terms schematically of the form

$$
\begin{aligned}
& {\left[Z^{J} \mathcal{A}(\tau, Y)\right]\left[Z^{K} \mathcal{A}(\tau, Y)\right] B^{m}\left[C_{J, K}(\tau, x, Y) \phi^{2}(x)\right],} \\
& {\left[Z^{J} \mathcal{A}(\tau, Y)\right] B^{m}\left[C_{J, K}(\tau, x, Y) \phi(x) Z^{K} \Psi(\tau, x, Y)\right],} \\
& B^{m}\left[C_{J, K}(\tau, x, Y) Z^{J} \Psi(\tau, x, Y) Z^{K} \Psi(\tau, x, Y)\right]
\end{aligned}
$$

with $C_{J, K} \in \mathcal{E}^{0}$. In $(6)|J|+|K| \leq N^{\prime}$. By (6.1), Lemma 7.1 and Lemma 2.4 we have

$$
\|(6)\|_{L_{Y}^{2} W_{x}^{1, \frac{p}{p-1}}} \leq\left\|C_{J, K}(\tau, x, Y) \phi^{2}\right\|_{L_{Y}^{\infty} W_{x}^{m+1, \frac{p}{p-1}}}\left(\mu^{\prime}\right)^{2} \epsilon^{2} .
$$

Similarly $\|(8)\|_{L_{Y}^{2} W_{x}^{1,} \frac{p}{p-1}}$ is bounded by a sum, for $j+k \leq m+1$, for $|K|+k \leq N / 2$ and for $\frac{1}{r}=\frac{p-1}{p}-\frac{1}{p}$, and by Sobolev embedding in the second step,

$$
C\left\|Z^{J} B^{j} \Psi(\tau)\right\|_{L_{Y}^{\infty} L_{x}^{r}}\left\|Z^{K} B^{k} \Psi(\tau)\right\|_{L_{Y}^{2} L_{x}^{p}} \leq E_{(1)}^{\frac{1}{2}}(\tau, \Psi)\left\|Z^{K} B^{k} \Psi(\tau)\right\|_{L_{Y}^{2} L_{x}^{p}} .
$$


By (6.1) and Lemma 8.1, the latter $\leq C\left(\mu^{\prime}\right) \epsilon^{2} \tau^{2 C\left(\mu^{\prime}\right) \epsilon-\left(\frac{1}{2}-2 \delta\right)}$, which is bounded. A similar bound is obtained for (7), and thus we obtain (2).

Now we turn to the proof of $(4)$ of Lemma 11.2. $\mathcal{F}_{4}$ is a sum of terms of the form

$$
\frac{1}{T^{1+a}}\left(\partial_{T}^{b} Z^{J} \Sigma\right)\left(Z^{K} B^{m} \Psi_{T x}\right)
$$

with $|J|+|K| \leq|I|, b \leq 1$ and $a \geq 1$ if $b=0$. If $|J| \leq\left[\frac{N}{2}\right]$, then by Lemma 7.1

$$
\left\|Z^{J} \frac{1}{T}\left(\Sigma_{T}-\frac{\Sigma}{T}\right)\right\|_{\infty} \leq C\left(\mu^{\prime}\right) \epsilon T^{-\frac{3}{2}+\delta}
$$

while by Lemmas 2.4 and 8.6 and by $|K|+m+2 \leq N^{\prime}+2 \leq N-1$

$\left\|Z^{K} B^{m} \Psi_{T x}\right\|_{L_{Y}^{2} W_{x}^{1, \frac{p}{p-1}}} \leq C \tau^{\frac{1}{2}-\frac{1}{p}}\left(\left\|\Psi_{N^{\prime}+2}\right\|_{L_{Y}^{2} H_{x}^{1}}+\frac{C\left(\mu^{\prime}\right) \epsilon}{\tau^{2}}\right) \leq C \tau^{\frac{1}{2}-\frac{1}{p}+C\left(\mu^{\prime}\right) \epsilon} C\left(\mu^{\prime}\right) \epsilon$.

As a consequence the desired estimate follows from the upper bound

$$
\int_{T_{0}}^{T} \frac{(9)}{(T-\tau)^{\frac{1}{2}-\frac{1}{p}}} \leq C\left(\mu^{\prime}\right) \epsilon \int_{T_{0}}^{T} \frac{1}{(T-\tau)^{\frac{1}{2}-\frac{1}{p}}} \frac{d \tau}{\tau^{1+\frac{1}{p}-2 \delta}} .
$$

If in $(9)|J|>\left[\frac{N}{2}\right]$, then by $(6.1)$

$$
\left\|Z^{J} \frac{1}{T}\left(\Sigma_{T}-\frac{\Sigma}{T}\right)\right\|_{2} \leq C\left(\mu^{\prime}\right) \epsilon T^{-\frac{3}{2}+\delta},
$$

while by Lemma 8.6 and Sobolev embedding

$$
\left\|Z^{K} B^{m} \Psi_{T x}\right\|_{L_{Y}^{\infty} W_{x}^{1, \frac{p}{p-1}}} \leq C \tau^{\frac{1}{2}-\frac{1}{p}}\left(\left\|\Psi_{N}\right\|_{L_{Y}^{2} L_{x}^{2}}+\frac{C\left(\mu^{\prime}\right) \epsilon}{\tau^{2}}\right) \leq C \tau^{\frac{1}{2}-\frac{1}{p}+C\left(\mu^{\prime}\right) \epsilon} C\left(\mu^{\prime}\right) \epsilon .
$$

We again obtain (10).

Along with the $L_{Y}^{2} L_{x}^{p}$ estimate, in (6.1) we also have low energy estimates for $\Psi$. We have:

Lemma 11.3. Assume (6.1). There is a fixed constant $C$ and a constant $\epsilon_{0}\left(\mu^{\prime}\right)$ such that if $\epsilon \in] 0, \epsilon_{0}\left(\mu^{\prime}\right)\left[\right.$ and $T \in\left[T_{0}, T^{*}[\right.$ we have

$$
\left\|\partial_{T} \Psi_{N^{\prime}}(T)\right\|_{L_{Y}^{2} H_{x}^{1}}+\left\|\Psi_{N^{\prime}}(T)\right\|_{L_{Y}^{2} H_{x}^{2}} \leq 2 \epsilon .
$$

We apply $B^{m} Z^{I}$ to formula (9.1) for $m+|I| \leq N^{\prime}+1$ and $m \geq 1$ :

$$
\begin{aligned}
& \left(B^{m} Z^{I} \Psi\right)_{T T}+B^{2} B^{m} Z^{I} \Psi+r_{x T} \partial_{x} B^{m} Z^{I} \Psi_{T} \\
& =-Z^{I} \frac{1}{T} B^{m} F_{2}(\Psi, \mathcal{A})-Z^{I} \frac{1}{T^{2}} B^{m} R_{\Psi} \\
& -\left[Z^{I}, r_{x T}\right] B^{m} \Psi_{x T}-r_{x T}\left[B^{m}, \partial_{x}\right] Z^{I} \Psi_{T} .
\end{aligned}
$$

We have the following Claim:

Claim. We have

$$
\left\|r h s(11.1)+Z^{I} \frac{1}{T} B^{m} 3 \operatorname{th} \phi^{2}(x) \mathcal{A}^{2}\right\|_{L_{x Y}^{2}} \leq \frac{C\left(\mu^{\prime}\right)}{T^{\frac{9}{8}}} \epsilon^{2} .
$$

Assume the Claim. For $D(T)=\left\|\left(B^{m} Z^{I} \Psi\right)_{T}\right\|_{L_{Y}^{2} L_{x}^{2}}^{2}+\left\|B^{m+1} Z^{I} \Psi\right\|_{L_{Y}^{2} L_{x}^{2}}^{2}$ by the Claim we get for $\psi(x)=3 \operatorname{th} \phi^{2}(x)$

$$
\frac{1}{2} \frac{d}{d T} D(T) \leq D^{\frac{1}{2}}(T) \frac{C\left(\mu^{\prime}\right) \epsilon^{2}}{T^{\frac{9}{8}}}-\left\langle\left(B^{m} Z^{I} \Psi\right)_{T}, Z^{I} \frac{1}{T} B^{m} \psi \mathcal{A}^{2}\right\rangle_{L_{x Y}^{2}} .
$$


If on an interval $\left[T_{0}, T_{1}\left[\right.\right.$ we have $D(T) \leq 8 \epsilon^{2}$, then for $\left.\epsilon \in\right] 0, \epsilon\left(\mu^{\prime}\right)[$ we have

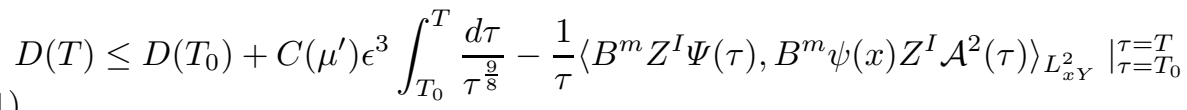

$$
+\int_{T_{0}}^{T}\left\langle B^{m} Z^{I} \Psi(\tau), B^{m} \psi(x) \partial_{T} Z^{I} \frac{1}{\tau} \mathcal{A}^{2}(\tau)\right\rangle_{L_{x Y}^{2}} .
$$

Now we claim

$$
\left|\left\langle B^{m} Z^{I} \Psi(T), B^{m} \psi(x) \partial_{T} Z^{I} \frac{1}{T} \mathcal{A}^{2}(T)\right\rangle_{L_{x Y}^{2}}\right| \leq \frac{C\left(\mu^{\prime}\right) \epsilon^{3}}{T^{\frac{5}{4}}} .
$$

By (6.1) we have $\left\|\partial_{T} Z^{I} \frac{1}{T} \mathcal{A}^{2}(T)\right\|_{2} \leq \frac{C\left(\mu^{\prime}\right) \epsilon^{2}}{T}$. (2) will follow from $\left\|B^{m} Z^{I} \Psi\right\|_{L_{Y}^{2} L_{x}^{p}} \leq$ $C\left(\mu^{\prime}\right) T^{-\frac{1}{4}} \epsilon$. Recall $m+|I| \leq N^{\prime}+1$ and $m \geq 1$. By interpolation for $Y$ fixed

$$
\left\|B \Psi_{N^{\prime}}\right\|_{L_{x}^{p}} \leq C_{1}\left\|\Psi_{N^{\prime}}\right\|_{L_{x}^{p}}^{\frac{N-N^{\prime}-2}{N-1}}\left\|\Psi_{N-1}\right\|_{L_{x}^{p}}^{\frac{1}{N-N^{\prime}-1}} \leq C_{2}\left\|\Psi_{N^{\prime}}\right\|_{L_{x}^{p}}^{\frac{N-N^{\prime}-2}{N-1}}\left\|\Psi_{N}\right\|_{L_{x}^{2}}^{\frac{1}{N-N^{\prime}-1}}
$$

where we use Sobolev embedding. Then by (6.1) and Lemma 8.1

$$
\begin{aligned}
& \left\|B \Psi_{N^{\prime}}\right\|_{L_{Y}^{2} L_{x}^{p}} \leq C_{2}\left\|\Psi_{N^{\prime}}\right\|_{L_{Y}^{2} L_{x}^{p}}^{\frac{N-N^{\prime}-2}{N-1}}\left\|\Psi_{N}\right\|_{L_{x Y}^{2}}^{\frac{1}{N-N^{\prime}-1}} \\
& \leq C\left(\mu^{\prime}\right) \epsilon T^{\frac{C\left(\mu^{\prime}\right) \epsilon}{N-N^{\prime}-1}-\frac{N-N^{\prime}-2}{N-N^{\prime}-1}\left(\frac{1}{2}-2 \delta\right)} \leq C\left(\mu^{\prime}\right) \epsilon T^{\tilde{C}\left(\mu^{\prime}\right) \epsilon-\frac{6}{7}\left(\frac{1}{2}-2 \delta\right)} .
\end{aligned}
$$

From (3) we get (2). Entering the information in (1), we get $D(T) \leq \epsilon^{2}+$ $C\left(\mu^{\prime}\right) \epsilon^{2}\left(T_{0}^{-\frac{1}{8}}+\epsilon\right)$ for some fixed function $C\left(\mu^{\prime}\right)$. Since $T_{0}$ can be thought large and $\epsilon>0$ small, we conclude that $D(T) \leq 4 \epsilon^{2}$ for $T \in\left[T_{0}, T_{1}\right]$. So we have proved that for any $T_{1}<T^{*}, D(T) \leq 8 \epsilon^{2}$ in $\left[T_{0}, T_{1}\right]$ implies $D(T) \leq 4 \epsilon^{2}$ in $\left[T_{0}, T_{1}\right]$. Hence we conclude $D(T) \leq 4 \epsilon^{2}$ in $\left[T_{0}, T^{*}[\right.$.

We now prove the Claim. By Sobolev embedding, by (7.3) and by $m+|I| \leq N^{\prime}+1$ with $m \geq 1$ for the first inequality, and by (6.1) and Lemma 8.1 for the second,

$$
\left\|\left[Z^{I}, r_{x T}\right] B^{m} \Psi_{x T}\right\|_{2} \leq \frac{1}{T}\left(\left\|\partial_{T} \Sigma_{N^{\prime}}\right\|_{2}+\frac{1}{T}\left\|\Sigma_{N^{\prime}}\right\|_{2}\right)\left\|\Psi_{N}\right\|_{L^{2}} \leq C\left(\mu^{\prime}\right) \epsilon^{2} T^{C\left(\mu^{\prime}\right) \epsilon+\delta-\frac{3}{2}} .
$$

The rhs is bounded by $C\left(\mu^{\prime}\right) T^{-\frac{9}{8}} \epsilon^{2}$.

By Lemma 7.1 , by the fact that $\left[B^{m}, \partial_{x}\right]$ is a pseudodifferential operator of order $m$ and by Lemma 8.1 ,

$$
\frac{1}{T}\left\|\left(\Sigma_{T}-\frac{\Sigma}{T}\right)\right\|_{\infty}\left\|\left[B^{m}, \partial_{x}\right] Z^{I} \Psi_{T}\right\|_{2} \leq \frac{C\left(\mu^{\prime}\right) \epsilon}{T^{1+\frac{1}{2}-\delta}}\|\Psi\|_{H^{N}} \leq \frac{C\left(\mu^{\prime}\right) \epsilon^{2}}{T^{1+\frac{1}{2}-\delta-C\left(\mu^{\prime}\right) \epsilon}} .
$$

By Lemma 9.2 , which we have yet to prove, $\left\|Z^{I} \frac{1}{T^{2}} B^{m} R_{\Psi}\right\|_{2} \leq C\left(\mu^{\prime}\right) \epsilon^{2} T^{-\frac{9}{8}}$.

By $F_{2}(\Psi, \mathcal{A})-3 \operatorname{th} \phi^{2}(x) \mathcal{A}^{2}=6 \operatorname{th} \phi(x) \mathcal{A} \Psi+3 \operatorname{th}(x) \Psi^{2}$, we claim

$$
\left\|Z^{I} \frac{1}{T} B^{m}\left[F_{2}(\Psi, \mathcal{A})-3 \operatorname{th} \phi^{2}(x) \mathcal{A}^{2}\right]\right\|_{2} \leq \frac{C\left(\mu^{\prime}\right)}{T^{\frac{9}{8}}} \epsilon^{2} .
$$

To check (4) observe that we need to bound a combination of $\Psi^{2}$ and of $\mathcal{A} \Psi$. We have $\left\|\left(\Psi^{2}\right)_{N^{\prime}+1}\right\|_{2} \leq C\left\|\Psi_{N^{\prime}}\right\|_{L_{Y}^{2} L_{x}^{p}}\left\|\Psi_{N}\right\|_{2} \leq C\left(\mu^{\prime}\right) T^{-\frac{1}{2}+2 \delta+C\left(\mu^{\prime}\right) \epsilon} \epsilon^{2}$ by (6.1), Hölder inequality, Sobolev embedding and Lemma 8.1. Next, we recall that $m+|I| \leq N^{\prime}+1$ with $m \geq 1$. Hence it is enough to bound

$$
\left\|B(\psi(x) \mathcal{A} \Psi)_{N^{\prime}}\right\|_{2} \leq C\left\|\mathcal{A}_{N}\right\|_{2}\left\|(1+B) \Psi_{N^{\prime}}\right\|_{L_{Y}^{2} L_{x}^{p}}
$$


By Lemma 8.1 we have $\left\|\mathcal{A}_{N}\right\|_{2} \leq C\left(\mu^{\prime}\right) T^{C\left(\mu^{\prime}\right) \epsilon} \epsilon$. By (3) and by (6.1) we have

$$
\left\|(1+B) \Psi_{N^{\prime}}\right\|_{L_{Y}^{2} L_{x}^{p}} \leq\left\|\Psi_{N^{\prime}}\right\|_{L_{Y}^{2} L_{x}^{p}}+\left\|B \Psi_{N^{\prime}}\right\|_{L_{Y}^{2} L_{x}^{p}} \leq C\left(\mu^{\prime}\right) \epsilon T^{\tilde{C}\left(\mu^{\prime}\right) \epsilon-\frac{6}{7}\left(\frac{1}{2}-2 \delta\right)} .
$$

\section{$\S 12$. Estimates FOR $\Sigma$}

In Klainerman's classical proof of dispersion of solutions of the zero mass wave equation (see texts [Ho], [So]) the Morawetz vectorfield takes a central role. Notice though that in these treatments dimension is at least 3 . With this remark in mind, we recall for $|I| \leq N^{\prime}$ equation $P Z^{I} \Sigma=\operatorname{rhs}(8.3)$. We then consider

$$
\sinh (R) \mathcal{K}\left(Z^{I} \Sigma\right) P Z^{I} \Sigma=\mathcal{K}\left(Z^{I} \Sigma\right) \operatorname{rhs}(8.3)
$$

and set, in the notation of Lemma 5.4,

$$
\mathcal{E}_{1}\left(T, Z^{I} \Sigma\right)=\iint d R d \theta \frac{\mathcal{P}^{0}}{\cosh (R)} .
$$

Then we have:

Lemma 12.1. Assume (6.1). Then, for $\epsilon_{0}$ small enough and for $\left.\epsilon \in\right] 0, \epsilon_{0}[$, for $|I| \leq N^{\prime}$ there is a fixed $C$ such that

$$
\frac{d}{d T} \mathcal{E}_{1}\left(T, Z^{I} \Sigma\right) \leq\left(C\left(\mu^{\prime}\right) \epsilon+C\right) \epsilon T^{-\frac{1}{2}+\delta} \mathcal{E}_{1}^{\frac{1}{2}}\left(T, Z^{I} \Sigma\right)+\frac{1}{T} \mathcal{E}_{1}\left(T, Z^{I} \Sigma\right) .
$$

Let us assume for the moment Lemma 12.1. By Gronwall inequality and by (6.1) we get:

Lemma 12.2. Assume (6.1). Then, there are a fixed $C$ and an $\epsilon_{0}$ small enough such that for $\epsilon \in] 0, \epsilon_{0}\left[\right.$, for $|I| \leq N^{\prime}$ we have

$$
\mathcal{E}_{1}^{\frac{1}{2}}\left(T, Z^{I} \Sigma\right) \leq C T^{\frac{1}{2}+\delta} \epsilon .
$$

In particular, for $|I| \leq N^{\prime}$,

$$
\begin{aligned}
& \left\|\frac{1}{\sinh (R)} \partial_{\theta} Z^{I} \Sigma\right\|_{L_{Y}^{2}} \leq C T^{\frac{1}{2}+\delta} \epsilon, \\
& \left\|T \partial_{T} Z^{I} \Sigma+\tanh (R) \partial_{R} Z^{I} \Sigma\right\|_{L_{Y}^{2}} \leq C T^{\frac{1}{2}+\delta} \epsilon .
\end{aligned}
$$

Notice that (1) can be used to get (6.2) for $\frac{1}{T \sinh (R)} \partial_{\theta} \Sigma$ but that (2) is not enough for $\partial_{T} \Sigma$ and for $\frac{\partial_{R}}{T} \Sigma$. For this reason we introduce

$$
\mathcal{E}_{2}\left(T, Z^{I} \Sigma\right)=\frac{1}{2} \iint d \theta d R \sinh (R)\left[T^{2}\left(\partial_{T} Z^{I} \Sigma\right)^{2}+\left(\partial_{R} Z^{I} \Sigma\right)^{2}+\frac{\left(\partial_{\theta} Z^{I} \Sigma\right)^{2}}{\sinh ^{2}(R)}\right] .
$$

Then we claim:

Lemma 12.3. We have for a fixed $C_{1}, \frac{d}{d T} \mathcal{E}_{2}\left(T, Z^{I} \Sigma\right) \leq$

$$
\left(C\left(\mu^{\prime}\right) \epsilon+C_{1}\right) \epsilon T^{-\frac{1}{2}+\delta} \mathcal{E}_{2}^{\frac{1}{2}}\left(T, Z^{I} \Sigma\right)+\frac{1}{T} \iint d \theta d R \sinh (R)\left(T \partial_{T} Z^{I} \Sigma\right)^{2} .
$$

We postpone the proof. As a consequence of Lemmas 12.2 and 12.3 we obtain:

Lemma 12.4. For $|I| \leq N^{\prime}$ and a fixed constant $\tilde{A}$, we have

$$
\begin{gathered}
\left\|T \partial_{T} Z^{I} \Sigma\right\|_{L_{Y}^{2}} \leq \tilde{A} T^{\frac{1}{2}+\delta} \epsilon, \\
\left\|\partial_{R} Z^{I} \Sigma\right\|_{L_{Y}^{2}} \leq \tilde{A} T^{\frac{1}{2}+\delta} \epsilon .
\end{gathered}
$$


Proof. Notice that $\mathcal{E}_{2}$ is defined with a factor $1 / 2$, so the proof does not follow immediately from Lemma 12.3 and Gronwall inequality, so we use Lemma 12.2. We express $\left[T_{0}, T^{*}\left[\right.\right.$ as a union of intervals $\left[T_{1}, T_{2}\left[\subseteq\left[T_{0}, T^{*}[\right.\right.\right.$ such that we have one of the following alternatives:

(1) $\forall T \in\left[T_{1}, T_{2}\left[\right.\right.$ we have $\left\|\partial_{R} Z^{I} \Sigma\right\|_{L_{Y}^{2}}^{2} \leq \frac{1-2 \delta}{1+2 \delta}\left\|T \partial_{T} Z^{I} \Sigma\right\|_{L_{Y}^{2}}^{2}$;

(2) $\forall T \in\left[T_{1}, T_{2}\left[\right.\right.$ we have $\left\|\partial_{R} Z^{I} \Sigma\right\|_{L_{Y}^{2}}^{2} \geq \frac{1-2 \delta}{1+2 \delta}\left\|T \partial_{T} Z^{I} \Sigma\right\|_{L_{Y}^{2}}^{2}$.

The union can be taken maximal, in the sense that intervals of type (1) and (2) alternate. For $\left[T_{1}, T_{2}\right.$ [ as in (1), from (2) in Lemma 12.3 we conclude that Lemma 12.4 holds in $\left[T_{1}, T_{2}\left[\right.\right.$ for $\tilde{A}=\tilde{B} \doteqdot C(1-\sqrt{1-2 \delta} / \sqrt{1+2 \delta})^{-1}, C$ of Lemma 12.2 and $\tilde{B}$ defined by the equality. For $\left[T_{1}, T_{2}[\right.$ as in $(2)$, then

$$
\left\|T \partial_{T} Z^{I} \Sigma\right\|_{2}^{2} \leq \frac{1+2 \delta}{2}\left(\left\|T \partial_{T} Z^{I} \Sigma\right\|_{2}^{2}+\left\|\partial_{R} Z^{I} \Sigma\right\|_{2}^{2}\right) .
$$

By Lemma 12.3, $\forall T \in\left[T_{1}, T_{2}[\right.$ we have:

$$
\frac{d}{d T} \mathcal{E}_{2}\left(T, Z^{I} \Sigma\right) \leq\left(C\left(\mu^{\prime}\right) \epsilon+C_{1}\right) \epsilon T^{-\frac{1}{2}+\delta} \mathcal{E}_{2}^{\frac{1}{2}}\left(T, Z^{I} \Sigma\right)+\frac{1}{T}(1+2 \delta) \mathcal{E}_{2}\left(T, Z^{I} \Sigma\right)
$$

and $\frac{d}{d T} \mathcal{E}_{2}^{\frac{1}{2}}\left(T, Z^{I} \Sigma\right) \leq \frac{1}{2}\left(C\left(\mu^{\prime}\right) \epsilon+C_{1}\right) \epsilon T^{-\frac{1}{2}+\delta}+\frac{1+2 \delta}{2 T} \mathcal{E}_{2}^{\frac{1}{2}}\left(T, Z^{I} \Sigma\right)$. By Gronwall we get $\mathcal{E}_{2}^{\frac{1}{2}}\left(T, Z^{I} \Sigma\right) \leq\left(\frac{T}{T_{1}}\right)^{\frac{1}{2}+\delta} \mathcal{E}_{2}^{\frac{1}{2}}\left(T_{1}, Z^{I} \Sigma\right)+\left(C\left(\mu^{\prime}\right) \epsilon^{2}+C_{1} \epsilon\right) T^{\frac{1}{2}+\delta}$. Since $T_{1}$ is the endpoint of an interval of type $(1)$, we have $\mathcal{E}_{2}^{\frac{1}{2}}\left(T_{1}, Z^{I} \Sigma\right) \leq \tilde{B} T_{1}^{\frac{1}{2}+\delta} \epsilon$. Then $\mathcal{E}_{2}^{\frac{1}{2}}\left(T, Z^{I} \Sigma\right) \leq\left(\frac{T}{T_{1}}\right)^{\frac{1}{2}+\delta} \tilde{B} T_{1}^{\frac{1}{2}+\delta} \epsilon+\left(C\left(\mu^{\prime}\right) \epsilon^{2}+C_{1} \epsilon\right) T^{\frac{1}{2}+\delta}$. For $\epsilon$ small, the latter gives Lemma 12.4 with $\tilde{A}=\tilde{B}+2 C_{1}$.

What is now left is the proof of Lemmas 12.2 and 12.3. By Lemma 5.5 we have

$$
\begin{aligned}
\frac{d}{d T} \mathcal{E}_{1}\left(T, Z^{I} \Sigma\right) & \leq\left\|\left(T \partial_{T}+\tanh R \partial_{R}\right) Z^{I} \Sigma\right\|_{L_{Y}^{2}} T\|\operatorname{rhs}(8.3)\|_{L_{Y}^{2}} \\
& -\frac{1}{T} \iint d R d \theta \frac{T \tanh (R)}{\cosh (R)} \mathcal{P}^{1} .
\end{aligned}
$$

By Lemma 5.6 we have, since the divergence terms in $\partial_{R}$ and $\partial_{\theta}$ disappear and by definition of $\mathcal{E}_{2}$,

$$
\frac{d}{d T} \mathcal{E}_{2}\left(T, Z^{I} \Sigma\right) \leq 2 \mathcal{E}_{2}^{\frac{1}{2}}\left(T, Z^{I} \Sigma\right) T\|\operatorname{rhs}(8.3)\|_{L_{Y}^{2}}+\frac{1}{T} \iint d \theta d R \sinh (R)\left(T \partial_{T} Z^{I} \Sigma\right)^{2} .
$$

We now have:

Lemma 12.5. Let $|I| \leq N^{\prime}$. Then we have

$$
\|r h s(8.3)\|_{2} \leq\left(C\left(\mu^{\prime}\right) \epsilon+C\right) \epsilon T^{-\frac{3}{2}+\delta} .
$$

Notice that Lemma 12.3 follows immediately from Lemma 12.5, while Lemma 12.2 is the consequence of the following two claims, after whose proof we start the proof of Lemma 12.5.

Claim 1. We have

$$
-T \frac{\tanh (R)}{\cosh (R)} \mathcal{P}^{1} \leq \frac{\mathcal{P}^{0}}{\cosh (R)}
$$


Indeed

$$
\begin{aligned}
& -\frac{\mathcal{P}^{1}}{\cosh (R)} \leq \frac{1}{2 T} \sinh (R) \\
& \times\left[\tanh (R)\left(T \partial_{T} Z^{I} \Sigma\right)^{2}+2 T \partial_{T} Z^{I} \Sigma \partial_{R} Z^{I} \Sigma+\tanh R\left(\partial_{R} Z^{I} \Sigma\right)^{2}\right]
\end{aligned}
$$

and

$$
\begin{aligned}
& \frac{\mathcal{P}^{0}}{\cosh (R)} \geq \frac{1}{2} \tanh (R) \cosh (R) \\
& \times\left[\left(T \partial_{T} Z^{I} \Sigma\right)^{2}+2 T \tanh (R) \partial_{T} Z^{I} \Sigma \partial_{R} Z^{I} \Sigma+\left(\partial_{R} Z^{I} \Sigma\right)^{2}\right] .
\end{aligned}
$$

From the definition of $\mathcal{P}^{0}$ we obtain:

Claim 2. We have

$$
\sinh (R)\left(T \partial_{T} Z^{I} \Sigma+\tanh (R) \partial_{R} Z^{I} \Sigma\right)^{2} \leq 2 \mathcal{P}^{0} / \cosh (R) .
$$

Proof of Lemma 12.5. First of all, Lemmas 7.10 and 8.1 give us $\left\|\left[Z^{I}, P\right] \Sigma\right\|_{2} \leq$ $C T^{-2+C\left(\mu^{\prime}\right) \epsilon} \epsilon$. By (8.8) in Lemma 8.5, Lemma 12.5 will hold if $\left\|Z^{I} \frac{\mathcal{A}}{T}\langle\Psi, \psi\rangle\right\|_{2} \leq$ $\frac{C\left(\mu^{\prime}\right) \epsilon^{2}}{T^{\frac{3}{2}-\delta}}$. The last inequality is a consequence of the following refinement of (8.6), which gives us a $\delta$ gain in the decay of $\Psi$ : for $|I| \leq N^{\prime}$ and $\psi(x)$ rapidly decreasing, we have

$$
\left\|Z^{I}\langle\Psi, \psi\rangle\right\|_{2} \leq C\left(\mu^{\prime}\right) \epsilon T^{-\frac{1}{2}+\delta} .
$$

(12.1) is crucial in our argument. Indeed when we estimate $\Sigma$ the exponents are tight. A decay $T^{-\frac{1}{2}+2 \delta}$ in (12.1) would lead to a decay $T^{-\frac{1}{2}+2 \delta}$ for $\Sigma$, with a disastrous feedback effect on the other estimates, and also on (12.1). So let us prove (12.1) assuming (6.1). Using the notation in $\S 11$ we write

$$
Z^{I}\langle\Psi, \psi\rangle=\sum_{j=1}^{4}\left\langle Z^{I} \Psi_{j}, \psi\right\rangle
$$

The terms corresponding to $j \neq 4$ satisfy (12.1) by Lemma 11.2. Indeed it is for $j=4$ that we need to gain a $T^{-\delta}$. We have

$$
\left\langle Z^{I} \Psi_{4}, \psi\right\rangle=-2 \int_{T_{0}}^{T} Z^{I}\left[\left\langle\Psi_{T x}(\tau), \frac{\sin (T-\tau) B}{B} \psi\right\rangle\left(\Sigma_{T}-\frac{\Sigma}{\tau}\right) \frac{1}{\tau}\right] d \tau .
$$

Ignoring the -2 , this is a sum of terms of the form

$$
\int_{T_{0}}^{T}\left\langle Z^{I^{\prime}} \Psi_{T x}(\tau), \frac{\sin (T-\tau) B}{B} \psi\right\rangle Z^{I^{\prime \prime}}\left(\Sigma_{T}-\frac{\Sigma}{\tau}\right) \frac{d \tau}{\tau}
$$

with $\left|I^{\prime}\right|+\left|I^{\prime \prime}\right|=|I|$. We bound pointwise

$$
|(1)| \leq C \int_{T_{0}}^{T}\left\|Z^{I^{\prime}} \Psi_{T x}(\tau)\right\|_{L_{x}^{1}}(T-\tau)^{-\frac{1}{2}}\left|Z^{I^{\prime \prime}} \frac{1}{\tau}\left(\Sigma_{T}-\frac{\Sigma}{\tau}\right)\right| d \tau .
$$

Since $\left|I^{\prime}\right|+\left|I^{\prime \prime}\right| \leq N^{\prime}$, by Lemma 8.6 and by (6.1) we have

$$
\|(1)\|_{L_{Y}^{2}} \leq C \int_{T_{0}}^{T}\left(\left\|\Psi_{T x}(\tau)\right\|_{H_{Y}^{N^{\prime}} L_{x}^{2}}+\frac{C \epsilon}{\tau^{2}}\right)(T-\tau)^{-\frac{1}{2}} \tau^{-\frac{1}{2}}\left(\left\|\partial_{T} \Sigma_{N^{\prime}}\right\|_{2}+\frac{1}{\tau}\left\|\Sigma_{N^{\prime}}\right\|_{2}\right) d \tau .
$$


By (6.1) and Lemma 11.3 we conclude that

$$
\|(2)\|_{L_{Y}^{2}} \leq \frac{C\left(\mu^{\prime}\right)}{\delta} \epsilon^{2} T^{-\frac{1}{2}+\delta} .
$$

In this way we conclude Lemma 12.5 .

\section{§13. Closure of the inequalities}

We conclude that (6.2) is a consequence of Lemmas 10.2, 11.1, 11.3, 12.2 and 12.4 .

\section{§14. Proofs of Lemmas 9.2 And 9.3}

Recall

$$
\begin{aligned}
& R_{\Psi}=\Delta_{h y p} \Psi+T \mathcal{Q}_{0}(\Sigma, \Sigma) P_{c} \operatorname{th}^{\prime \prime}-2 T \mathcal{Q}_{0}(\Sigma, \mathcal{A}) P_{c} \phi^{\prime}+\mathcal{A} \mathcal{Q}_{0}(\Sigma, \Sigma) P_{c} \phi^{\prime \prime} \\
& +P_{c} F_{3}(\Psi, \mathcal{A})+Q_{0}(\Sigma, \Sigma) P_{c} \Psi_{x x}+T P_{c}\left(\Psi_{x}+\mathcal{A} \phi^{\prime}\right) P \Sigma-T_{\alpha \neq T}^{2} r_{x \alpha} \Psi_{x \alpha}
\end{aligned}
$$

By (4) in Lemma 8.4 we have

$$
\left\|Z^{I} B^{m+1} T \mathcal{Q}_{0}(\Sigma, \mathcal{A}) P_{c} \phi^{\prime}\right\|_{L_{x Y}^{2} \cap L_{Y}^{2} L_{x}^{\frac{p}{p-1}}} \leq C\left(\mu^{\prime}\right) \epsilon^{2} T^{\frac{3}{4}}
$$

We will prove for $|I|+m \leq N^{\prime}$

$$
\left\|Z^{I} B^{m+1}\left[R_{\Psi}+2 T \mathcal{Q}_{0}(\Sigma, \mathcal{A}) P_{c} \phi^{\prime}\right]\right\|_{2} \leq\left(C\left(\mu^{\prime}\right) \epsilon+C\right) \epsilon T^{\frac{3}{4}-\frac{1}{2}+\frac{1}{p}}
$$

which is stronger than Lemma 9.2. $\left\|Z^{I} \Delta_{h y p} \Psi\right\|_{2} \leq C T^{C\left(\mu^{\prime}\right) \epsilon} \epsilon$ by (9.3). For the other terms in the first line, except the already discussed $-2 T \mathcal{Q}_{0}(\Sigma, \mathcal{A}) P_{c} \phi^{\prime}$, we have: $\left\|Z^{I} T \mathcal{Q}_{0}(\Sigma, \Sigma)\right\|_{2} \leq C\left(\mu^{\prime}\right) \epsilon^{2} T^{2 \delta}$ by (6) in Lemma 8.4; $\left\|Z^{I}\left[\mathcal{A} \mathcal{Q}_{0}(\Sigma, \Sigma)\right]\right\|_{2} \leq$ $C\left(\mu^{\prime}\right) \epsilon^{2} T^{2 \delta-1}$ by (8) in Lemma 8.4. By (1) in Lemma 7.4 and by Lemma 8.1 we have $\left\|Z^{I} B^{m} F_{j}(\Psi, \mathcal{A})\right\|_{2} \leq C\left(\mu^{\prime}\right) \epsilon^{2} T^{C\left(\mu^{\prime}\right) \epsilon}$. We have $\left\|Z^{I} B^{m} Q_{0}(\Sigma, \Sigma) P_{c} \Psi_{x x}\right\|_{2} \leq$ $C\left(\mu^{\prime}\right) \epsilon^{3} T^{2 \delta+C\left(\mu^{\prime}\right) \epsilon-1}$ by (6) in Lemma 8.4 and Lemma 8.1. By Lemmas 8.1 and 8.5 we have $\left\|Z^{I} B^{m} T P_{c}\left(\Psi_{x}+\mathcal{A} \phi^{\prime}\right) P \Sigma\right\|_{2} \leq C\left(\mu^{\prime}\right) \epsilon^{3} T^{2 \delta+C\left(\mu^{\prime}\right) \epsilon-\frac{1}{2}}$. Next, recall

$$
r_{x x}=\frac{1}{T^{2}} \mathcal{Q}_{0}(\Sigma, \Sigma) \quad, \quad r_{x R}=r_{R x}=\frac{2}{T^{3}} \Sigma_{R}, r_{x Y_{j}}=r_{Y_{j} x}=\frac{2 \Sigma_{\theta}}{T^{3} \sinh (R)} a_{j}(Y)
$$

where $a_{1}(Y)=-\frac{Y_{2}}{\sinh (R)}$ and $a_{2}(Y)=\frac{Y_{1}}{\sinh (R)}$. Then by (6) in Lemma 8.4 and by Lemma 8.1 we have $\left\|Z^{I} B^{m} r_{x x} \Psi_{x x}\right\|_{2} \leq C\left(\mu^{\prime}\right) \epsilon^{3} T^{2 \delta+C\left(\mu^{\prime}\right) \epsilon-1}$; by (6.1) and by Lemma 8.1 we have in the other cases $\left\|Z^{I} B^{m} r_{x \alpha} \Psi_{x \alpha}\right\|_{2} \leq C\left(\mu^{\prime}\right) \epsilon^{2} T^{\delta-\frac{1}{2}+C\left(\mu^{\prime}\right) \epsilon}$.

Turning to Lemma 9.3, we have proved (14.1) for each single term in the formula for $R_{\Psi}$ except for $2 T \mathcal{Q}_{0}(\Sigma, \mathcal{A}) P_{c} \phi^{\prime}$. For each term with a cutoff in $x$, the estimate translates automatically in the estimate required for Lemma 9.3. For each term linear in $\Psi$ we can use Lemma 8.6. For the remaining term, by (2) in Lemma 7.4 we get

$$
\left\|Z^{I} B^{m+1}\left(\Psi^{3}\right)\right\|_{L_{Y}^{2} L_{x}^{\frac{p}{p-1}}} \leq C\left(\mu^{\prime}\right) \epsilon\left(\epsilon+T^{-\frac{1}{2}+2 \delta}\left\|(\Psi, \mathcal{A})_{N}\right\|_{2}\right)\left\|(\Psi, \mathcal{A})_{N}\right\|_{2} .
$$

The desired bound follows from Lemma 8.1.

\section{ACKNowledgments}

I wish to thank Professor Fabio Zanolin for his hospitality at the University of Udine. 


\section{REFERENCES}

[AS] Ablowitz, Segur, Solitons and the Inverse Scattering Transform, SIAM Studies in Appl. Math, SIAM, 1981. MR642018 (84a:35251)

[D] Delort, Existence globale et comportamente asymptotique pour l'equation de Klein Gordon quasilineaire a donnees petites en dimension 1, Ann. Scient. Ec. Norm. Sup. 34 (2001), $1-61$.

[DFX] Delort, Fang, Xue, Global existence of small solutions for quadratic quasilinear Klein Gordon systems in two space dimensions, preprint.

[K] Klainerman, Global existence of small amplitude solutions to nonlinear Klein-Gordon equations in four space-time dimension, Comm. Pure Appl. Math. 38 (1985), 631-641. MR803252 (87e:35080)

[Ka] Kapitula, Multidimensional stability of planar travelling waves, Trans. Amer. Math. Soc. 349 (1998), 257-269. MR1360225 (97d:35104)

[Ko] Kosecki, The Unit Condition and Global Existence for a Class of Nonlinear Klein-Gordon Equation, Jour. Diff. Eq 100 (1992), 257-268. MR1194810 (93k:35178)

[GK] Goldman, Krivchenkov, Problems in quantum mechanics, Dover.

[GP] Georgiev, Popivanov, Global solution to the two-dimensional Klein-Gordon equation, Comm. Part. Diff. Eq. 16 (1991), 941-995. MR1116850 (92g:35140)

[GSS] Grillakis, Shatah, Strauss, Stability theory of solitary waves in the presence of symmetry, I, Jour. Funct. Anal. 74 (1987), 160-197. MR901236 (88g:35169)

[H] Henry, Geometric theory of semilinear parabolic equations, Lecture Notes in Mathematics, 840, Springer, 1981. MR610244 (83j:35084)

[HPW] Henry, Perez, Wreszinski, Stability theory for solitary-wave solutions of scalar field equations, Comm. Math. Phys. 85 (1982), 351-361. MR678151 (83m:35131)

[Ho] Hörmander, Lectures on Nonlinear Hyperbolic Differential Equations, Springer, 1996.

[LX] Levermore, Xin, Multidimensional stability of traveling waves in a bistable reactiondiffusion equation. II, Comm. Part. Diff. Eq. 17 (1992), 1901-1924. MR1194744 (94c:35105)

[OTT] Ozawa, Tsutaya, Tsutsumi, Global existence and asymptotic behavior of solutions for the Klein-Gordon equations with quadratic nonlinearity in two space dimension, Math. Z. 222 (1996), 341-362. MR1400196 (97e:35112)

[Sh1] Shatah, Global existence of small solutions to nonlinear evolution equations, J. Diff. Eq. 46 (1982), 409-425. MR681231 (84g:35036)

[Sh2] , Normal forms and quadratic nonlinear Klein Gordon equations, Comm. Pure Appl. Math. 38 (1985), 685-696. MR0803256 (87b:35160)

[So] Sogge, Lectures on nonlinear wave equations, Monographs in Analysis, II, International Press, 1995. MR1715192 (2000g:35153)

[T] Taylor, Pseudodifferential operators, Princeton Math. Series, 34, Princeton Un. Press, 1981. MR618463 (82i:35172)

[We] Weder, The $W^{k, p}$ continuity of the Schrödinger wave operators on the line, Comm. Math. Phys. 208 (1999), 507-520. MR1729096 (2001c:34178)

[X] Xin, Multidimensional stability of traveling waves in a bistable reaction-diffusion equation, Comm. PDE 17 (1992), 1889-189.

Dipartimento di Scienze e Metodi per l'Ingegneria, Università di Modena e Reggio Emilia, Padiglione Morseldi, via Amendola 2, Reggio Emilia 42100, Italy

E-mail address: cuccagna.scipio@unimore.it 